\title{
Quadboost: A Scalable Concurrent Quadtree
}

\author{
Keren Zhou, Guangming Tan, Wei Zhou
}

\begin{abstract}
Building concurrent spatial trees is more complicated than binary search trees since a space hierarchy should be preserved during modifications. We present a non-blocking quadtree-quadboost-that supports concurrent insert, remove, move, and contain operations. To increase its concurrency, we propose a decoupling approach that separates physical adjustment from logical removal within the remove operation. In addition, we design a continuous find mechanism to reduce its search cost. The move operation combines the searches for different keys together and modifies different positions with atomicity. The experimental results show that quadboost scales well on a multi-core system with 32 hardware threads. More than that, it outperforms existing concurrent trees in retrieving two-dimensional keys with up to $109 \%$ improvement when the number of threads is large. The move operation proved to perform better than the best-known algorithm, with up to $47 \%$.
\end{abstract}

Index Terms-Concurrent Data Structures, Quadtree, Continuous Find, Decoupling, LCA

\section{INTRODUCTION}

$\mathrm{M}$ ULTI-CORE processors are currently the universal computing engine in computer systems. Therefore, it is urgent to develop data structures that provide an efficient and scalable multi-thread execution. At present, concurrent data structures [1], such as stacks, linked-lists, and queues have been extensively investigated. As a fundamental building block of many parallel programs, these concurrent data structures provide significant performance benefits [2], [3].

Recently, research on concurrent trees is focused on binary search trees (BSTs) |4], |5], |6], |7], [8], |9|, |10], which are at the heart of many tree-based algorithms. The concurrent paradigms of BSTs were extended to design concurrent spatial trees like R-Tree [11], [12]. However, there remains another unaddressed spatial tree-quadtree, which is widely used in applications for multi-dimensional data. For instance, spatial databases, like PostGIS [13], adopt octree, a three-dimensional variant of quadtree, to build spatial indexes. Video games apply quadtree to handle collision detection [14]. In image processing [15], quadtree is used to decompose pictures into separate regions.

There are different categories of quadtree according to the type of data a node represents, where two major types are region quadtree and point quadtree [16]. Point quadtree stores actual keys in each node, yet it is hard to design concurrent algorithms for point quadtree since an insert operation might involve re-balance issues, and a remove operation needs to re-insert the whole subtree under a removed node. The region quadtree divides a given region into several sub-regions, where internal nodes represent regions and leaf nodes store actual keys. Our work focuses on region quadtree for two reasons: (1) The shape of region quadtree is independent of insert/remove operations' order, which allow us to avoid complex re-balance rules and devise specific concurrent techniques for it. (2) Further, region quadtree could be regarded as a typical external tree that

- K.Zhou and G.Tan are with Institute of Computing Technology, Chinese Academy of Sciences.

- W.Zhou is with School of Software, Yunnan University. we can adjust existing concurrent techniques from BSTs. Therefore, we will refer to region quadtree as quadtree in the following context.

In this paper, we design a non-blocking quadtree, referred to as quadboost, that supports concurrent insert, remove, contain, and move operations. Our key contributions are as follows:

- We propose the first non-blocking quadtree. It records traversal paths, compresses Empty nodes if necessary, adopts a decoupling technique to increase the concurrency, and devises a continuous find mechanism to reduce the cost of retries induced by CAS failures.

- We design a lowest common ancestor (LCA) based move operation, which traverses a common path for two different keys and modifies two distinct nodes with atomicity.

- We prove the correctness of quadboost algorithms and evaluate them on a multi-core system. The experiments demonstrate that quadboost is highly efficient for concurrent updating at different contention levels.

The rest of this paper is organized as follows. In Section 2 , we overview some basic operations. Section 3 describes a simple CAS quadtree to motivate this work. Section 4 provides detailed algorithms for quadboost. We provide a sketch of correctness proof in Section 5. Experimental results are discussed in Section 6. Section 7 summarizes related works. Section 8 concludes the paper.

\section{Preliminary}

Quadtree can be considered as a dictionary for retrieving two-dimensional keys, where $\langle k e y X, k e y Y>$ is never duplicated. Figure 1 illustrates a sample quadtree and its corresponding region, where we use numbers to indicate keys. Labels on edges are routing directions-Southwest (sw), Northwest (nw), Southeast (se), and Northeast (ne). 
The right picture is a mapping of the quadtree on a twodimensional region, where keys are located according to their coordinates, and regions are divided by their corresponding width $(w)$ and height $(h)$. There are three types of nodes in the quadtree, which represent different regions in the right figure. Internal nodes are circles on the left figure, and each of them has four children which indicate four equal sub-regions on different directions. The root node is an Internal node, and it is the largest region. Leaf nodes and Empty nodes are located at the terminal of the quadtree; they indicate the smallest regions on the right figure. Leaf nodes are solid rectangles that store keys; they represent regions with the same numbers on the right figure. Empty nodes are dashed rectangles without any key.

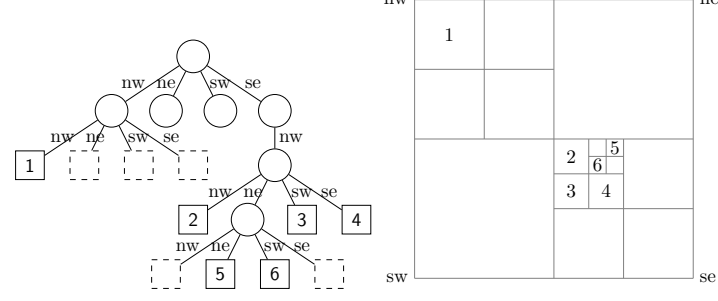

Fig. 1: A sample quadtree and its corresponding region

We describe the detailed structures of a quadtree in Figure 2 An Internal node maintains its four children and a two-dimensional routing structure- $<x, y, w, h>$, where $<x, y\rangle$ stands for the upper left coordinate and $\langle w, h\rangle$ are the width and height of the region. A Leaf node contains a key <keyX, key $Y>$ and its corresponding value. An Empty node does not have any field. To avoid some corner cases, we initially split the root node and its children to form two layers of dummy Internal nodes with a layer of Empty nodes at the terminal. Also, we present routing functions find and getQuadrant in the figure. For instance, if we have to locate key 1 in Figure 1 , we start with the root node and compare key 1 with its routing structures by getQuadrant. Then we reach its $n w$ and perform a comparison again. In the end, we find the terminal node that contains key 1 .

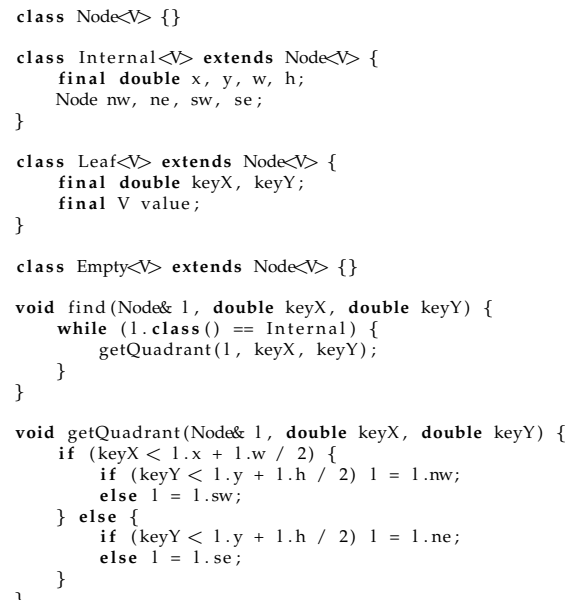

Fig. 2: Quadtree nodes and routing functions

There are four basic operations that rely on find:
1) insert(key, value) adds a node that contains key and value into a quadtree.

2) remove(key) deletes an existing node with key from a quadtree.

3) contain (key) checks whether key is in a quadtree.

4) move(oldKey, newKey) replaces an existing node with oldKey and value by a node with newKey and value that is not in a quadtree.

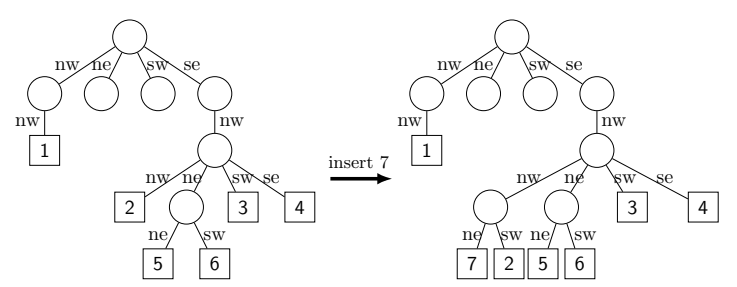

(a) Insert node 7 into the sample quadtree

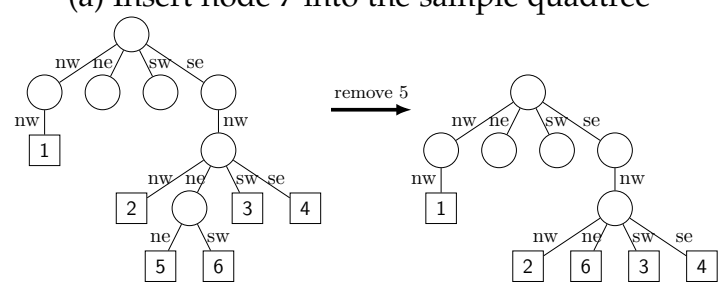

(b) Remove node 5 from the sample quadtree

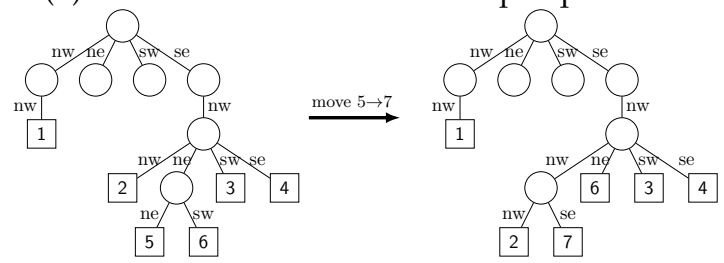

(c) Move the value of node 5 to node 7 from the sample quadtree

Fig. 3: Sample quadtree operations ${ }^{1}$

To insert a node, we first locate its position by calling find. If we find an Empty node at the terminal, we directly replace it by the node. Otherwise, before replacing the candidate node, we recursively divide a Leaf node into four corresponding sub-regions until the candidate region is Empty. Figure 3a illustrates a scenario of inserting node 7 (insert(7)) as a neighborhood of node 2 . The parent node is split, and node 7 is added on the ne direction. Likewise, to remove a node, we also begin by locating it and then erase it from a quadtree. Next, we check whether its parent contains a single Leaf node. If so, we record the node and traverse up until reaching a node that contains at least a Leaf node or the child of the root node. Finally, we use that node as a new parent and re-insert the recorded node as its child. Take Figure $3 b$ as an example, if we remove node 5 (remove(5)) from a quadtree, node 6 will be linked to the upper level. The move operation is a combination of the insert operation and the remove operation. It first removes the node with oldKey and then adds a new node with newKey into a quadtree. Consider the scenario in Figure 3c, after removing node 5 and inserting node 7 with the same value $($ move $(1,7))$, the new tree appears on the right part. Because contain(key) just checks whether the node returned by find has the same key, it does not need an extra explanation.

1. Empty nodes are not drawn 


\section{CAS QuAdTREE}

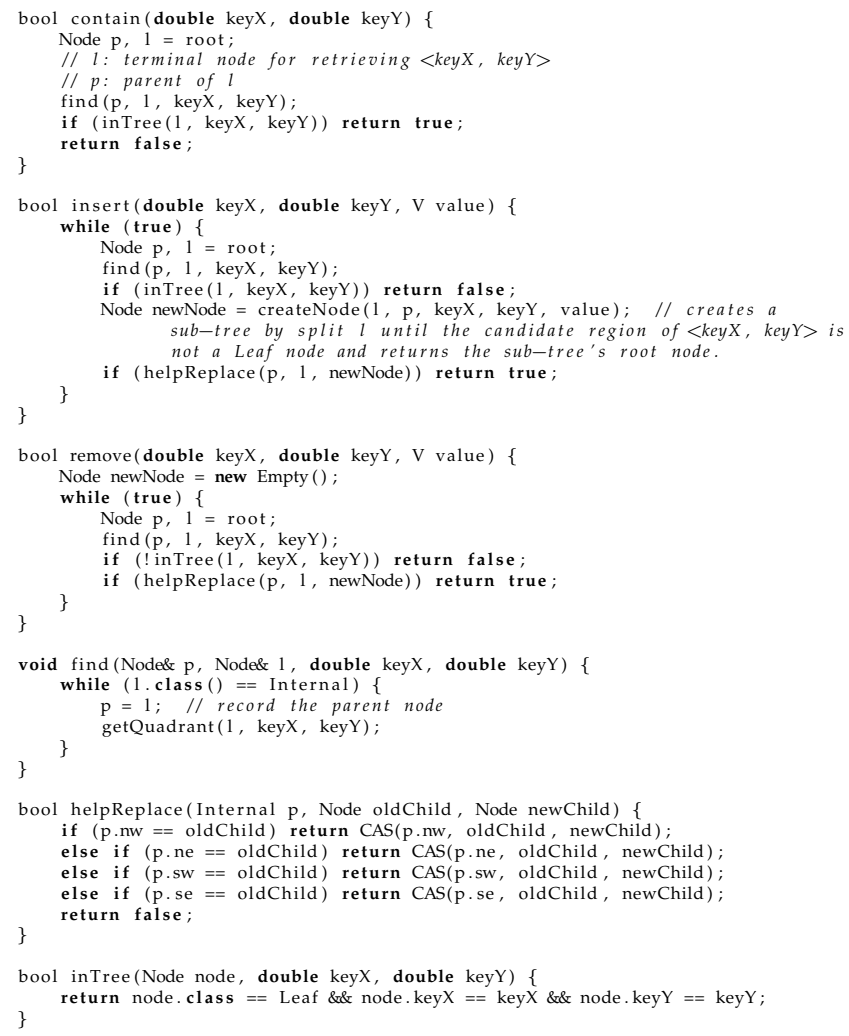

Fig. 4: CAS quadtree algorithm

There are a plenty of concurrent tree algorithms, yet a formal concurrent quadtree has not been studied. Intuitively, we can devise a concurrent quadtree by modifying the sequential algorithm described in Section 2 and adopting CAS instruction, which we name CAS quadtree. Figure 4 depicts CAS quadtree's algorithms. Both the insert operation and the remove operation follow such a paradigm: it starts by locating a terminal node; then, it checks whether the node satisfies some conditions. If conditions are not satisfied, it returns false. Otherwise, it tries to apply a single CAS to replace the terminal node by a new node. After a successful CAS, it returns true. Or else, it restarts locating a terminal node from the root node. For a insert operation, if $<$ key $\mathrm{X}, \mathrm{key} \mathrm{Y}>$ is not in the tree, it creates a corresponding sub-tree that contains < keyX, keyY $>$ and value (line 38. Then it uses the root of the sub-tree to replace the terminal node by a single CAS (line 39). To remove a node, it creates an Empty node at the beginning (line 43). If $<$ keyX, keyY $>$ is in the tree, it uses the Empty node to replace the terminal node (line 48). Different from the sequential algorithm, we do not adjust the structure as it involves several steps that cannot be implemented with atomicity. The algorithm is non-blocking. In other words, the algorithm provides a guaranteed whole progress, even if some threads starve.

However, CAS quadtree has several limitations. First, consider if there are a considerable proportion of remove operations. By applying the simple mechanism, we still have a large number of nodes in the quadtree because we substitute existing nodes with Empty nodes without structural

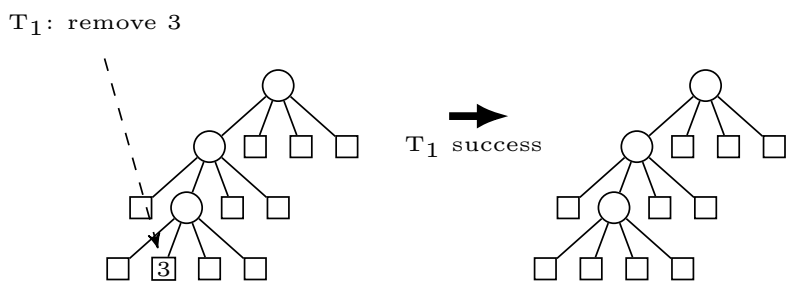

Fig. 5: Thread $\mathrm{T}_{1}$ intends to remove node 3 from quadtree. After its removal, there remains a chain of Empty nodes

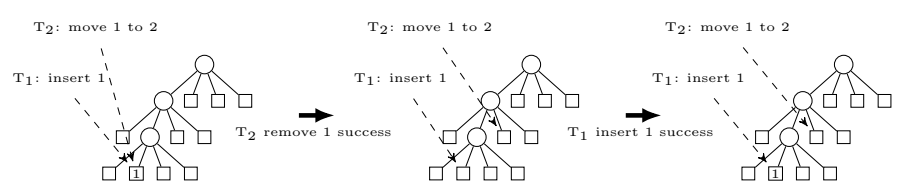

Fig. 6: An example of an incorrect move operation. Thread $\mathrm{T}_{1}$ intends to insert node 1 into a quadtree, and thread $\mathrm{T}_{2}$ plans to move the value from node 1 to node 2 . $T_{2}$ first successfully removes node 1 and then attempts to insert node 2 into the quadtree. In the interval node 1 is added back by $T_{1}$, but $T_{2}$ is not aware of the action and reports success.

adjustment. Figure 5 illustrates a detailed example showing that there remains a chain of Empty nodes. Hence, not only we have to traverse a long path to locate the terminal node for basic operations, but also a plenty of nodes are maintained in the memory.

Second, we cannot implement the move operation in the simple algorithm. There might be two different nodes in a quadtree that were under modifying. If we directly erase the node with oldKey and add the node with newKey, the move operation cannot be correctly linearized. Figure 6 shows such an incorrect move operation by combining an insert operation and a remove operation together.

Therefore, these drawbacks motivate us to develop a new concurrent algorithm to make the move operation correct and employ an efficient mechanism to compress nodes.

\section{Quadboost}

\subsection{Rationale}

In this section, we describe how to design quadboost algorithms to solve the two problems addressed in Section 3

To make the move operation correct, we should ensure that other threads know whether a terminal node is under moving. Hence, we attach an internal node with a separate object-Operation (op) to represent a node's state and record sufficient information to complete the operation. We instantiate the attachment behavior as a CAS and call it a flag operation. We design different ops for the insert operation, the remove operation and the move operation. The detailed description of structures and a state transition mechanism is presented in Section 4.2

To erase Empty nodes from a quadtree, we can apply a similar paradigm in the concurrent BST's removal [4] as shown in Figure 7a, which flags both the parent and the 
$\boldsymbol{r}$ - remove flag, $\boldsymbol{i}$ - insert flag

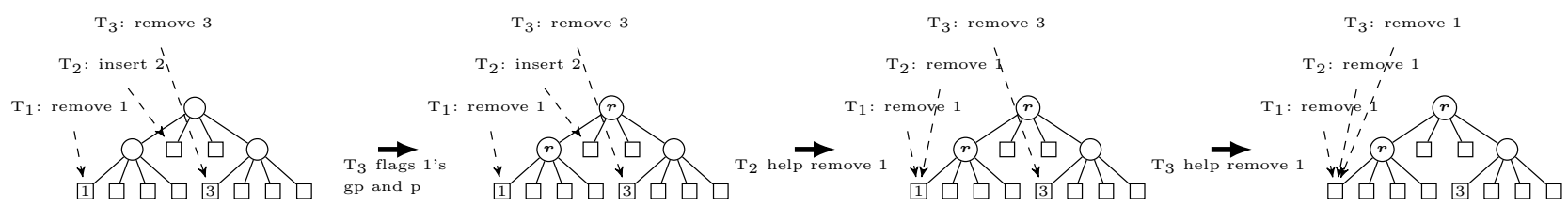

(a) Apply traditional removal
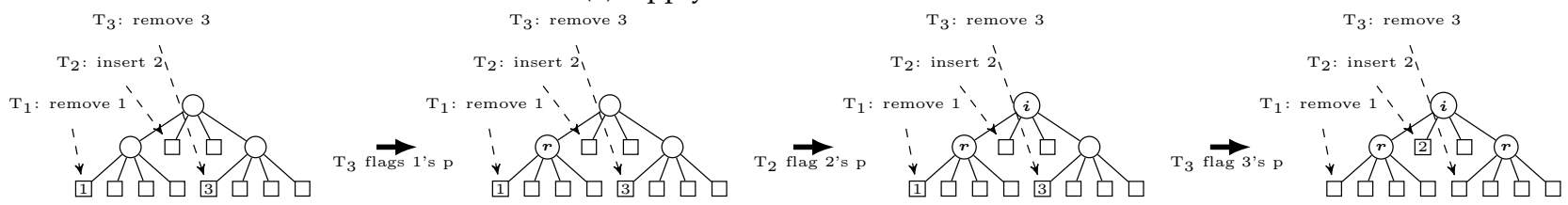

(b) Our decoupling approach

Fig. 7: At the beginning, three threads are performing different operations concurrently: (a) $T_{1}$ removes key 1 in the lower level. (b) $T_{2}$ inserts key 2 in the upper level. (c) $T_{3}$ removes key 3 in the lower level. Consider the scenario that $T_{1}$ precedes others threads, and then both 1's parent and grandparent will be flagged. Hence, $T_{2}$ and $T_{3}$ will help $T_{1}$ remove key 1 before restarting their operations. By applying our decoupling method, only the parent node should be flagged. Hence three threads could run without intervention.

grandparent of a terminal node. This mechanism mixes logical removal and physical removal together. Different from the BST's removal in which every time the parent node has to be adjusted, we only have to compress the parent when there is only a single Leaf child. Hence, we could separate the removal of a node and the adjustment of the structure into two phases. Meanwhile, we design an op-Compress to indicate a node is underlying the structural adjustment. Figure $7 \mathrm{~b}$ illustrates how this method increases concurrency: three threads that handle different ops could run in parallel. Further, for simplicity, we relax the adjustment condition to where the parent could be compressed if all children are Empty.

However, there is still a problem left after applying the above two methods. Recall the example in Figure 5 We flag the bottom Internal node to indicate that it should be compressed, but after replacing the bottom Internal node with an Empty node, it results in four Empty nodes in the last level. How do we remove a series of nodes from a quadtree in a bottom-up way? We record the entire traversal path from the root to a terminal node in a stack. Since the traversal path will be altered when a node is compressed, we only have to restart locating the terminal node from its parent if any flag operation other than the flag of Compress fails. This is called the continuous find mechanism.

\subsection{Structures and State Transitions}

As mentioned before, we add an Operation object to handle concurrency issues. Figure 8 shows the data structures of quadboost. Four sub-classes of Operation, including Substitute, Compress, Move, and Clean, describe all states in our algorithm. Substitute provides information on the insert operation and the remove operation that are designed to replace an existing node by a new node. Hence, we shall let other threads be aware of its parent, child, and a new node for substituting. Compress provides information for physical adjustment. We erase the parent node, previously connected to the grandparent, by swinging the link to an Empty node.

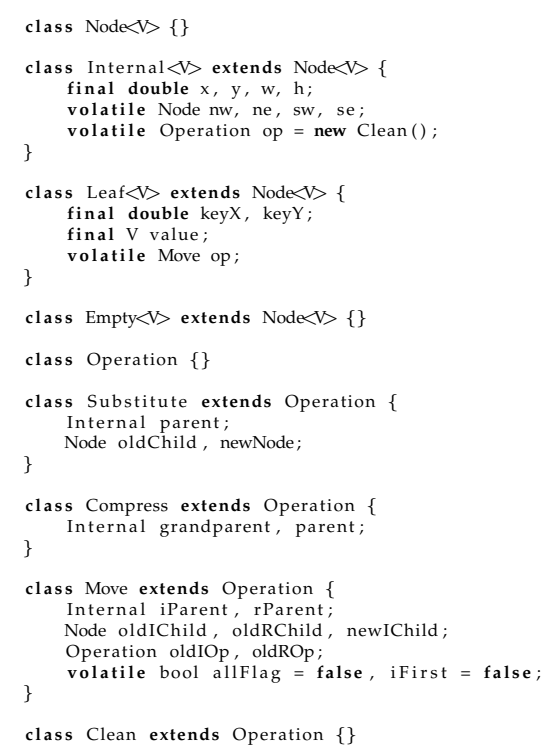

Fig. 8: quadboost structures

Move stores both oldKey's and newKey's terminal nodes, their parents, their parents' prior ops, and a new node. Moreover, we use a bool variable-allFlag to indicate whether two parents have been attached to a Move op or not. Another bool variable-iFirst is used to indicate the attaching order. For instance, if iFirst is true, iParent will be attached with a Move op before rParent. Clean means that there is no thread modifying the node. In contrast to Figure 4 the Internal class adds an op field to hold its state and related information. Note that a flag operation (CAS) is only applied on Internal nodes. We atomically set Move ops in Leaf nodes to linearize the move operation correctly.

Each basic operation, except for the contain operation, starts by changing an Internal node's op from Clean to other states. The three basic operations, therefore, generate a cor- 
responding state transition diagram which provides a highlevel description of our algorithm. After locating a terminal node, insert, remove, compress, and move transitions are executed as shown in Figure 9 In the figure, we specify the flag operation that restores an op to Clean as unflag, the flag operation that changes an op from Clean to Substitute in insert 102 as iflag, the flag operation that changes an op from Clean 10 to Substitute in remove as rflag, and the flag operation that changes an op from Clean to Compress as cflag. We describe how these transitions execute when a thread detects a state as follows:

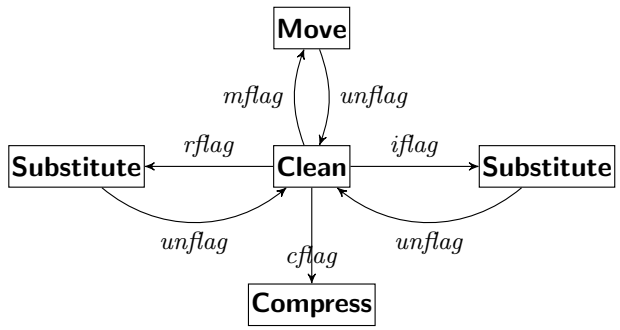

Fig. 9: State transition diagram

- Clean. For the insert transition, a thread constructs a new node and changes the parent's op to Substitute by iflag. For the remove transition, the thread creates an Empty node and changes the parent's op from Clean to Substitute by rflag. For the move transition, the thread flags both newKey's and oldKey's parents by mflag. For the compress transition, the thread uses a cflag ${ }_{14}^{142}$ operation to flag the parent if necessary.

- Substitute. The thread uses a CAS to change the existing node by a given node stored in the op. It then restores the parent's state to Clean by unflag.

- Move. The thread first determines the flag order ${ }^{15}$ by comparing oldKey's parent with newKey's parent. Suppose it flags newKey's parent first, it will flag old- ${ }_{153}^{153}$ Key's parent later. Then, the thread replaces oldKey's terminal with an Empty node and replaces newKey's terminal with a new node. Finally, it unflags their 156 parents' op to Clean in the reverse order.

- Compress. The thread erases the node from the tree so that it cannot be detected. Different than other states, the node with a Compress op cannot be set to Clean by unflag.

\subsection{Concurrent Algorithms}

Figure 10 reflects quadboost's insert and contain operations. Both of them start by a find process that locates a terminal node. The find operation (line 98 pushes Internal nodes into a stack (line 95) and keeps recording the parent node's op (line 96).

The contain operation executes in a similar way as CAS quadtree. It calls the find function to locate a terminal node (line 98). The op at line 96 and the stack at line 95 are created for a modular presentation. We can omit them in a real implementation.

In the insert operation, we create a stack at the beginning to record the traversal path (line 98) and a $p O p$ to record the parent node's op (line 96. After locating a terminal

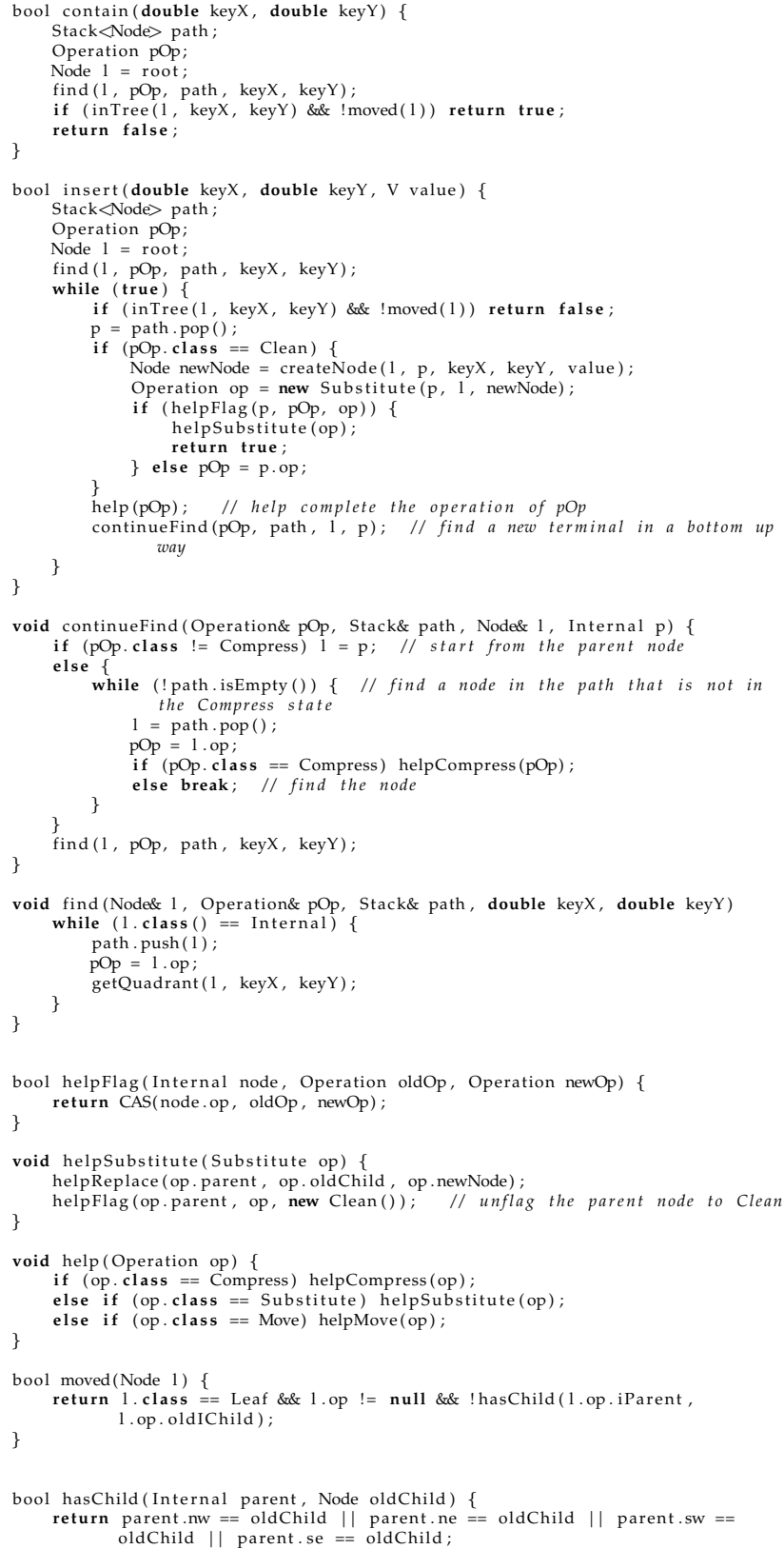

Fig. 10: quadboost insert and contain

node, we check whether the key of the node is in the tree and whether the node is moved at line 108 We will show the reason why we have to check whether a node is moved in Section 4.4. Then, we flag the parent of the terminal node before replacing it. In the next step, we call the helpSubstitute function at line 114. which first invokes the helpReplace function at line 145 to replace the terminal node and then unflag the parent node at line 146 If the flag operation fails, we update $p O p$ at line 116 and help it finish at line 118 . More than that, we have to execute the continueFind function to restart from the nearest parent. The continueFind function (line 122) pops nodes from the path until reaching a node whose op is not Compress. If the node's op is Compress, it helps the op finish. Or else, it breaks the loop and performs the find operation from the last node 
popped at line 132

The remove operation has a similar paradigm as the insert operation. It first locates a terminal node and checks whether the node is in the tree or moved. After that, it flags the parent node and replaces the terminal node with an Empty node. An extra step in the remove operation is the compress function at line 171 In the algorithm, we perform the compress function before the remove operation returns true. In this way, the linearization point of the remove operation belongs to the execution of itself, and extra adjustment induced by the compress function do affect the effectiveness. The compress function should examine three conditions before compressing the parent node. First, because the remove operation must return true, we do not compress nor help if the state of the parent is not Clean (line 182). Second, we check if the grandparent node $(g p)$ is the root (line 184) as we maintain two layers of dummy Internal nodes. At last, we check whether four children of a parent node are all Empty (line 186).

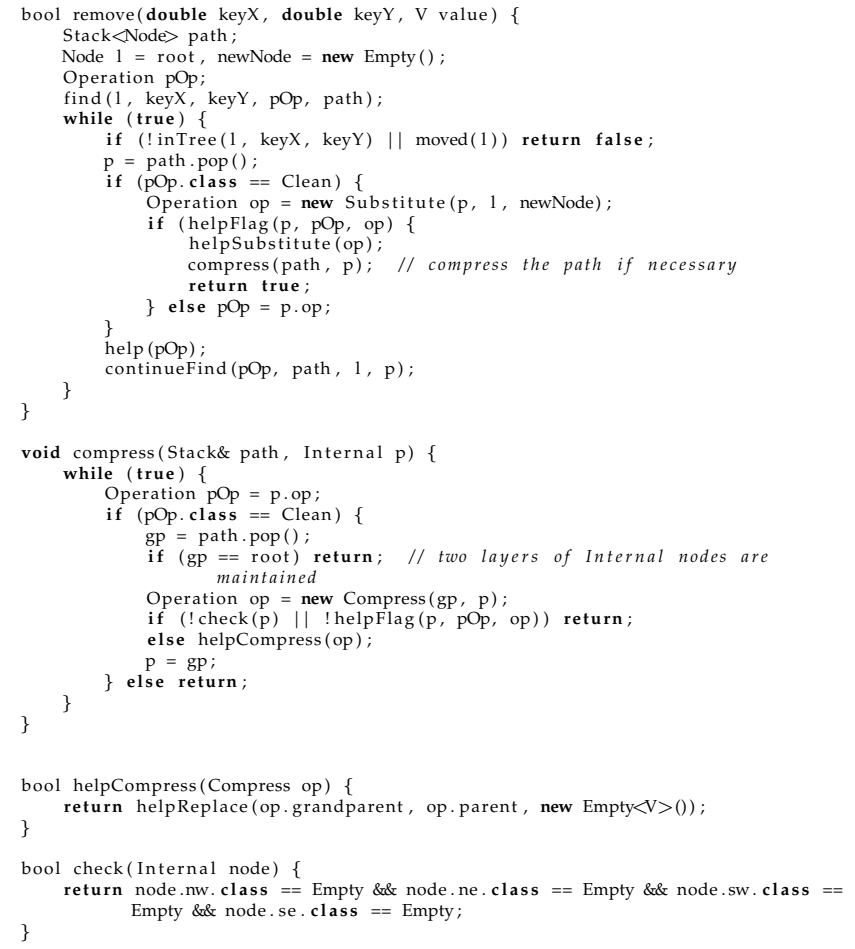

Fig. 11: quadboost remove

\subsection{LCA-based Move Operation}

Tree-based structures share a property that two nodes in the tree have a common path starting from the root, and the lowest node in the path is called the lowest common ancestor (LCA). Figure 12 demonstrates the LCA node of a quadtree by a concrete example. Based on the observation, our LCA-based move operation is defined to find two different terminal nodes sharing a common path, remove a node with oldKey, and insert a node with newKey.

Figures 13 and 14 present the algorithm of the move operation. In contrast with the insert operation and the remove operation, the move operation begins by calling $\boldsymbol{r}$ - remove path, $\boldsymbol{i}$ - insert path, $\boldsymbol{c}$ - common path

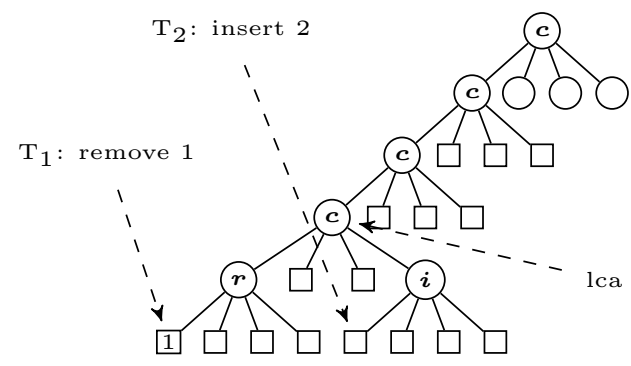

Fig. 12: Paths of two operations-insert node 2 and remove node 1 that share the LCA node

the findCommon function (line 212 that combines searches for two nodes together. The two searches share a common path that allows us to record them only once. We use two stacks to record nodes at line 199 The shared path and the remove path are pushed into rPath; and the insert path is pushed into $i$ Path. By doing this, we could avoid considering complicated corner cases in terms of node compressions in rPath. The findCommon function begins by reading a child node from the parent for oldKey and checks whether newKey is in the same direction (line 271). If not, it terminates the traversal, and searches for individual keys separately (line 282 and line 279). If oldKey is not in the tree or is moved, or if newKey is in the tree and not moved, it returns false.

The move operation then checks two parents' op (iOp and $r O p$ ) before flag operations. If neither of them is Clean (line 216 217), or $r p$ and $i p$ are the same but their ops are different (line 218), it starts the continueFindCommon function at line 243 which we will discuss later in the section. Otherwise, it creates a new node for inserting and an $o p$ to hold essential information for a CAS at line 220224 There are two specific cases. If two terminal nodes share a common parent, we directly call the helpMove function at line 228 Or else, to avoid live locks, we flag two nodes in a specific order. In our algorithm, we use the getSpatialOrder function at line 223 to compare $i p$ and $r p$ in the following order: $x \rightarrow y \rightarrow w$, where $x, y$, and $w$ are the fields of an Internal node. We prove that this method produces a unique order among all Internal nodes in a quadtree in appendix A

To start, the helpMove function flags a parent and checks whether both parents are successfully flagged at line 248 . 250 If both flag operations succeed, it sets allFlag to true at line 252. Note that we assign the op to oldRChild at line 253. letting other threads know whether the CAS on iParent has finished, which is the linearization point of the move operation. Because simultaneously oldRChild is aware that it is removed, it also explains why we should examine whether a node is moved. Then, we check whether two terminal nodes are the same. If so, we could combine two CASs into a single one. If not, we should remove oldKey's terminal and replace newKey's terminal. In the end, we reset the parents' op to Clean in the reverse order (line 261 267).

The continueFindCommon function is also invoked when one of the flag operations fails. If $r p$ cannot be flagged, it pops nodes from rPath until it reaches the LCA node (line 291) or a node's op is Compress (line 297). It then starts from the last popped node to search for a new terminal of 


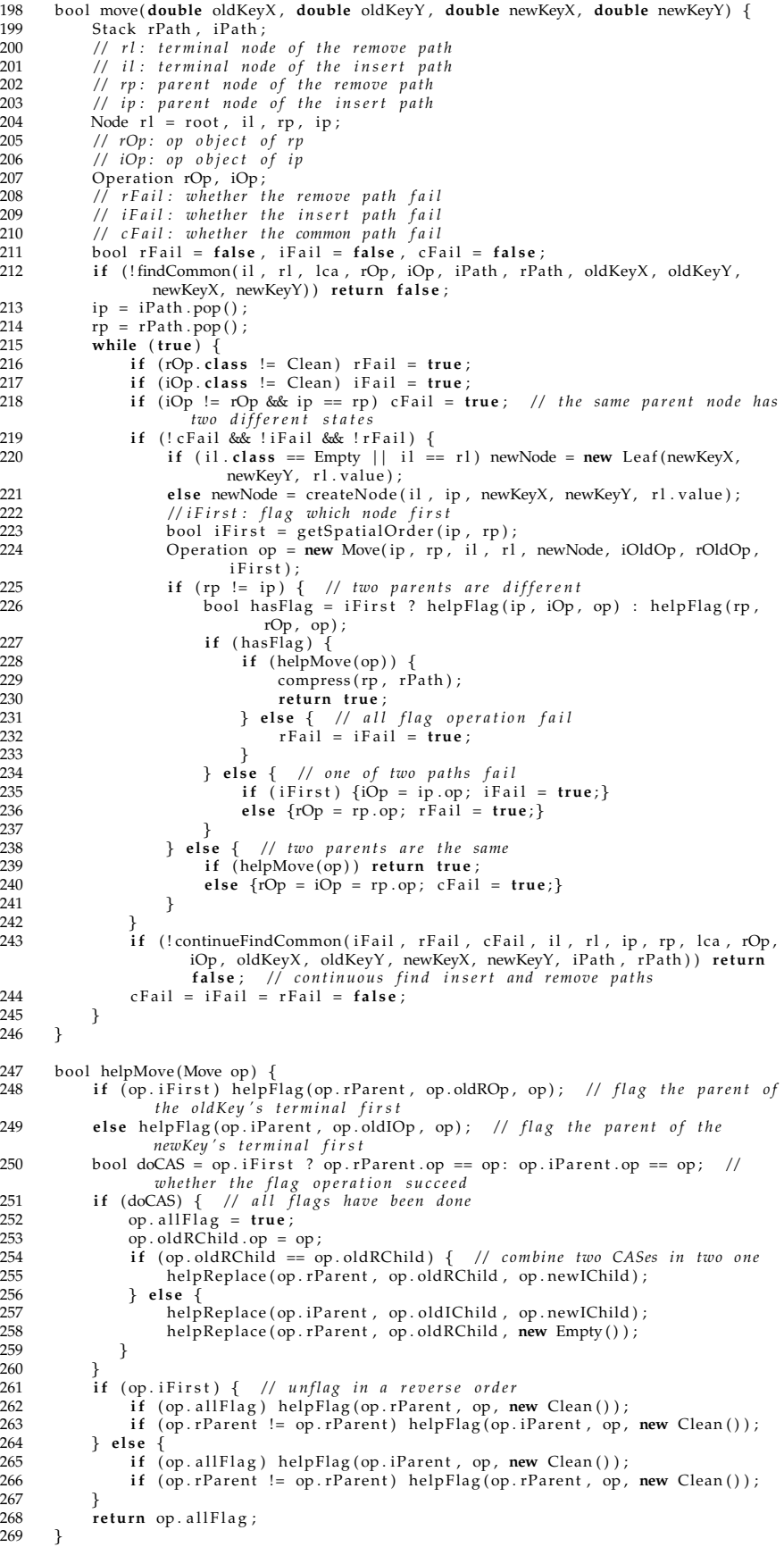

Fig. 13: quadboost move

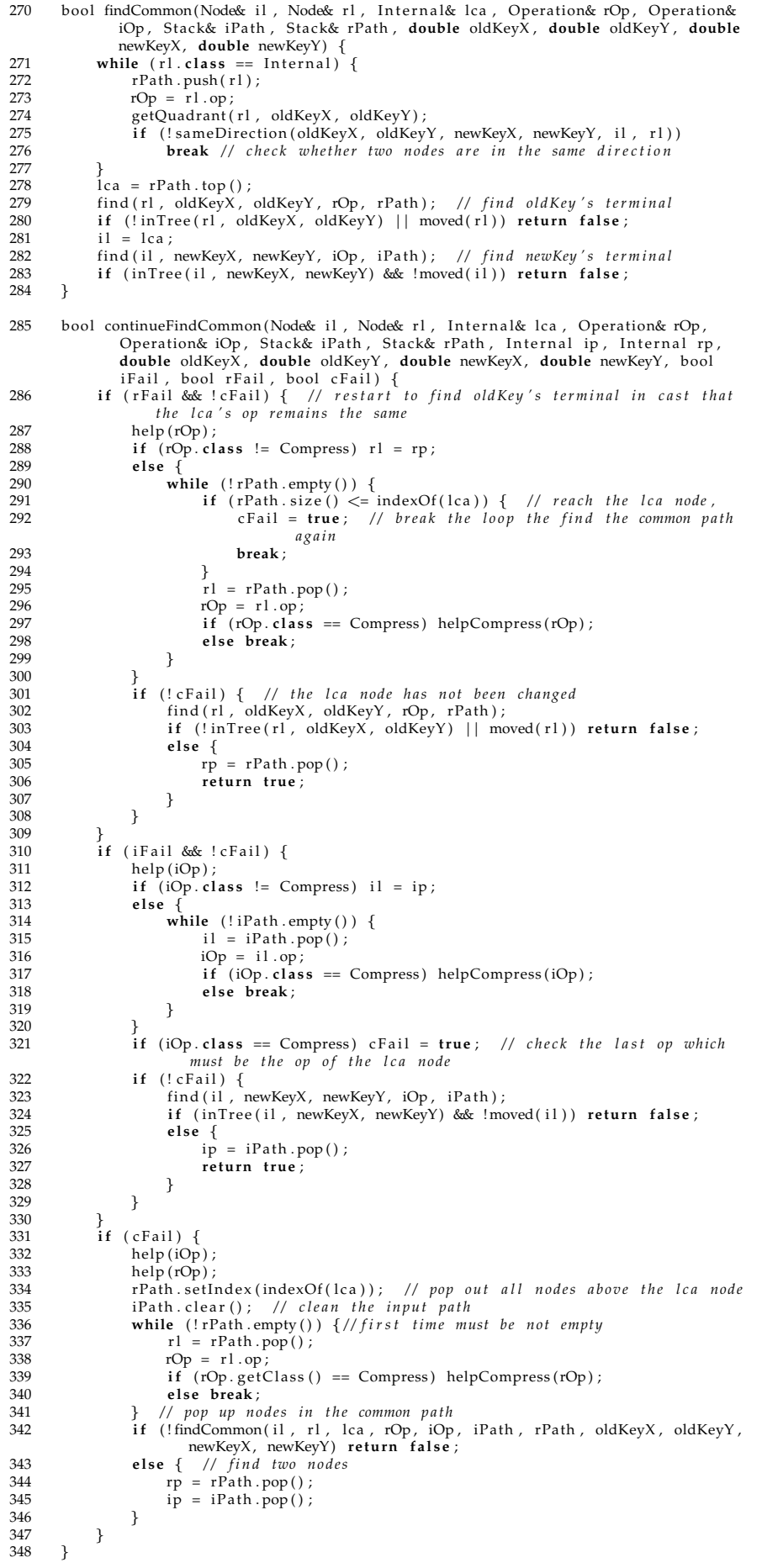

Fig. 14: quadboost findCommon and continueFindCommon

\subsection{One Parent Optimization}

oldKey at line 302. If ip cannot be flagged, it pops nodes from iPath until it is empty (line 315) or a node's op is not Compress (line 317). It again continues to search for newKey at line 323 If either $r$ Path has popped the LCA node or iPath is empty, it clears $i P a t h$ at line 335 and pops all nodes above the LCA node from rPath at line 334 . In the end, it calls the findCommon function to locate terminal nodes again at line 342 We prove that if oldKey's terminal and newKey's terminal share an LCA node, the common path will never be changed unless the LCA is altered.
In practice, we notice that pushing a whole stack during a traversal is highly expensive. When detecting a failed flag operation, we only have to restart from the parent of a terminal node because we do not change Internal nodes unless the compress function erases them from a quadtree. Thus, to reduce the pushing cost, we could only record the parent of the terminal node during a traversal.

Meanwhile, we have to change the continuous find 
mechanism. For the insert operation and the remove operation, encountering a Compress op, we straightforwardly restart from the root. For the move operation, if either oldKey's or newKey's parent is under compression, we restart from the LCA node. If the LCA node also has a Compress op, we restart from the root.

\section{Proof SKetch}

In this section, we prove that quadboost is both linearizable and non-blocking, and we propose a lengthy proof in appendix A

There are four kinds of basic operations in quadboost: the insert operation, the remove operation, the move operation, and the contain operation. Other functions are called subroutines, which are invoked by basic functions. The insert operation and the remove operation only modify one terminal node, whereas the move operation might operate two different terminals-one for inserting a node with newKey, the other for removing a node with oldKey. We call them newKey's terminal and oldKey's terminal, and we call their parents newKey's parent and oldKey's parent, respectively. We define snapshot $_{T_{i}}$ as the state of our quadtree at some time, $T_{i}$.

In our proofs, a CAS that changes a node's op is a flag operation and a CAS that changes a node's child is a replace operation. Specifically, we use iflag, rflag, mflag, and cflag to denote flag operations for the insert operation, the remove operation, the move operation, and the compress operation separately. Likewise, we use ireplace, rreplace, mreplace, and creplace for replace operations. Moreover, we specify a flag operation that attaches a Clean op on a node as an unflag operation.

First, we present some observations from quadboost. Then, we propose lemmas to show that our subroutines satisfy their pre-conditions and post-conditions because proofs on basic operations depend on these conditions. Next, we demonstrate that there are three categories of successful CAS transitions according to Figure 9 . We also derive some invariants of these transitions. Using above post-conditions of subroutines and invariants, we could demonstrate that a quadtree's structure is maintained during concurrent modifications. In following proofs, we show that quadboost is linearizable because it can be ordered equivalently as a sequential one by its linearization points. In the last part, we prove the non-blocking progress condition of quadboost.

Observation 1. The key field of a Leaf node is never changed. The op field of a Leaf node is initially null. The space information of an Internal node is never changed.

Observation 2. The root node is never changed.

Observation 3. A flag operation attaches an op on a Internal node.

Observation 4. The allFlag and iFirst field in Move are initially false, and they will never be set back after assigning to true.

Observation 5. If a Leaf node is moved, its op is set before the replace operation on op.iParent, which is before the replace operation on op.rParent.
Observation 6. The help function, the helpCompress function, the helpMove function, and the helpSubstitute function are not called in a mutual way. (If method A calls method $B$, and method $B$ also calls method $A$, we say $A$ and $B$ are called in a mutual way.)

\subsection{Basic Invariants}

We use find(keys) to denote a set of find operations: find, continueFind, findCommon, and continueFindCommon. The find function and the continueFind function return a tuple $\langle l, p O p, p a t h\rangle$. The findCommon function and the continueFindCommon function return two such tuples. We specify functions outside the while loop in the insert operation, the remove operation, and the move operation are at teration $_{0}$, and functions inside the while loop are at iteration $_{i}, 0<i$ ordered by their invocation sequence.

We suppose that find(keys) executes from a valid snapshot $_{T_{i}}$ to derive the following conditions. Proofs for conditions of other subroutines are included in appendix A

Lemma 1. The post-conditions of find(keys) returned at $T_{i}$, with tuples $\left\langle l^{k}, p O p^{k}, p a t h^{k}\right\rangle, 0 \leq k<|k e y s|$.

1) $l^{k}$ is a Leaf node or an Empty node.

2) At some $T_{i 1}<T_{i}$, the top node in path $h^{k}$ has contained $p O p^{k}$.

3) At some $T_{i 2}<T_{i}$, the top node in path $h^{k}$ has contained $l^{k}$.

4) If $p O p^{k}$ is read at $T_{i 1}$, and $l^{k}$ is read at $T_{i 2}$, then $T_{i 1}<$ $T_{i 2}<T_{i}$

5) For each node $n$ in the path ${ }^{k}$, size $\left(\right.$ path $\left.^{k}\right) \geq 2, n_{t}$ is on the top of $n_{t-1}$, and $n_{t}$ is on the direction $d \in$ $\{n w, n e, s w, s e\}$ of $n_{t-1}$ at $T_{i 1}<T_{i}$.

Based on these post-conditions, we show that each op created at $T_{i}$ store their corresponding information.

Lemma 2. For op created at $T_{i}$ :

1) If $o p$ is Substitute, op.parent has contained op.oldChild that is a Leaf node at $T_{i 1}<T_{i}$ from the results of find(keys) at the prior iteration.

2) If op is Compress, op.grandparent has contained op.parent that is an Internal node at $T_{i 1}<T_{i}$ from the results of find(keys) at the prior iteration.

3) If op is Move, op.iParent has contained op.oldIChild that is a Leaf node and op.oldIOp before $T_{i}$, and op.rParent has contained op.oldRChild that is a Leaf and op.oldROp before $T_{i}$ from the results of find(keys) at the prior iteration.

Though we have not presented details of the createNode function, our implementation could guarantee that it has following conditions.

Lemma 3. For createNode $(l, p$, newKey $X$, newKey $Y$, value $)$ that returns a new Node invoked at $T_{i}$, it has the post-condition:

1) The newNode returned is either a Leaf node with newKey and value, or a sub-tree that contains both l.key node and newKey node with the same parent.

Using prior conditions, we derive some invariants during concurrent executions and prove that there are three 
kinds of successful CAS transitions. We put successful flag operations that attach ops on nodes at the beginning of each CAS transition. We say every successive replace operations that read $o p$ belongs to it and follows the flag operations.

Let flag $_{0}, f l a g_{1}, \ldots$, flag $_{n}$ be a sequence of successful flag operations. flag $g_{i}$ reads $p O p_{i}$ and attaches $o p_{i}$. replace ${ }_{i}$ and $u_{n f l a g}$ read $o p_{i}$ and follows it. Therefore, we say flag $g_{i}$

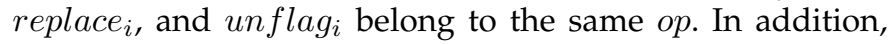
if there are more than one replace operation belongs to the same $o p_{i}$, we denote them as replace ${ }_{i}^{0}$, replace $_{i}^{1}, \ldots$, replace $_{i}^{n}$ ordered by their successful sequence. Similarly, if there is more than one flag operation that belonging to $o p_{i}$ on different nodes, we denote them as $f l a g_{i}^{0}, \operatorname{flag}_{i}^{1}, \ldots$, $\mathrm{flag}_{i}^{n}$. A similar notation is used for unflag $_{i}$.

The following lemmas prove the correct ordering of three different transitions.

Lemma 4. For a new node $n$ :

1) It is created with a Clean op.

2) rflag, iflag, mflag or cflag succeeds only if n's op is Clean.

3) unflag succeeds only if n's op is Substitute or Move.

4) Once n's op is Compress, its op will never be changed.

Lemma 5. For an Internal node $n$, it never reuses an op that has been set previously.

Lemma 6. replace ${ }_{i}^{k}$ will not occur before flag ${ }_{i}^{k}$ that belongs to the same op that has been done.

Lemma 7. The flag $\rightarrow$ replace $\rightarrow$ unflag transition occurs when rflag $_{i}$, iflag or $_{\text {mflag }}$ succeeds, and it has following properties:

1) replace $_{i}$ never occurs before flag . $_{\text {. }}$

2) flag $_{i}^{k}, 0 \leq k<\mid$ flag $_{i} \mid$ is the first successful flag operation on op $p_{i}$.parent ${ }^{k}$ after $T_{i 1}$ when $p O p_{i}^{k}$ is read.

3) replace $_{i}^{k}, 0 \leq k<\mid$ replace $_{i} \mid$ is the first successful replace operation on ${ }_{0} p_{i}$.parent ${ }^{k}$ after $T_{i 2}$ when op . oldChild $^{k}$ is read.

4) replace $_{i}^{k}, 0 \leq k<\mid$ replace $_{i} \mid$ is the first successful replace operation on op $p_{i}$.parent ${ }^{k}$ that belongs to $o p_{i}$.

5) unflag $_{i}^{k}, 0 \leq k<\mid$ unflag $_{i} \mid$ is the first successful unflag operation on op ${ }_{i}$.parent ${ }^{k}$ after flag fla $_{i}^{k}$.

6) There is no successful unflag operation that occurs before replace . $_{\text {. }}$.

7) The first replace operation on $o p_{i} \cdot$ parent ${ }^{k}$ that belongs to op $i$ must succeed.

Lemma 8. The flag $\rightarrow$ replace transition occurs only when cflag $_{i}$ succeeds, and it has following properties:

1) creplace $_{i}$ never occurs before cflag $_{i}$.

2) cflag $_{i}$ is the first successful flag operation on op $p_{i}$.parent after $T_{i 1}$ when $p O p_{i}$ is read.

3) creplace $_{i}$ is the first successful replace operation on

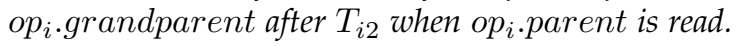

4) creplace $_{i}$ is the first successful replace operation on $o p_{i}$.grandparent that belongs to op .

5) There is no unflag operation after creplace . $_{\text {. }}$

6) The first replace operation on $o_{i}$.grandparent that belongs to op i must succeed.

Lemma 9. For the flag $\rightarrow$ unflag transition, it only results from mflag (Suppose iFirst is false) when:
1) unflag $_{i}$ is the first successful unflag operation on $o p_{i}$. P Parent.

2) The first flag operation on $o p_{i} . i$ Parent must fail, and no later flag operation succeeds.

3) $o p_{i} . i$ Parent and $o p_{i} . r$ Parent are different.

Claim 1. There are three kinds of successful transitions belong to an op: (1) flag $\rightarrow$ replace $\rightarrow$ unflag, (2) flag $\rightarrow$ unflag, (3) flag $\rightarrow$ replace.

Then, we prove that a quadtree maintains its properties during concurrent modifications.

Definition 1. Our quadtree has the following properties:

1) Two layers of dummy Internal nodes are never changed.

2) An Internal node $n$ has four children located in the direction $d \in\{n w, n e, s w, s e\}$ according to their $\langle x, y, w, h\rangle$, or $\langle$ key $X, \operatorname{key} Y\rangle$.

For Internal nodes residing on four directions:

- $\quad n . n w . x=n . x, n . n w . y=n . y$;

- $\quad$ n.ne. $x=n . x+w / 2, n . n e . y=n . y$;

- $n . s w . x=n . x, n . s w . y=n . y+n . h / 2$;

- $\quad$ n.se. $x=n . x+w / 2, n . s e . y=n . y+h / 2$,

All children have their $w^{\prime}=n . w / 2, h^{\prime}=n . h / 2$. For Leaf nodes residing on four directions:

- $\quad n . x \leq n . n w . k e y X<n . x+n . w / 2$, $n . y \leq n . n w . k e y Y<n . y+n . h / 2$;

- n.x +n.w $/ 2 \leq n . n e . k e y X<n . x+n . w$, $n . y \leq n . n e . k e y Y<n . y+n . h / 2$;

- $\quad n . x \leq n . s w . k e y X<n . x+n . w / 2$, $n . y+n . h / 2 \leq n . s w . k e y Y<n . y+n . h$;

- $n . x+n . w / 2 \leq n . s e . k e y X<n . x+n . w$, $n . y+n . h / 2 \leq n . s e . k e y Y<n . y+n . h$.

To help clarify a quadtree's properties during concurrent executions, we define active set and inactive set for different kinds of nodes. For an Internal node or an Empty node, if it is reachable from the root in snapshot $_{T_{i}}$, it is active; otherwise, it is inactive. For a Leaf node, if it is reachable from the root in snapshot $_{T_{i}}$ and not moved, it is active; otherwise, it is inactive. We say a node $n$ is moved in snapshot $T_{i}$ if the function moved(n) returns true at $T_{i}$. We denote $\operatorname{path}\left(k_{\text {eeys }}^{k}\right), 0 \leq k<\mid$ replace $_{i} \mid$ as a stack of nodes pushed by find(keys) in a snapshot. We define physical_path $\left(\right.$ keys $\left.^{k}\right)$ to be the path for keys ${ }^{k}$ in snapshot $T_{i}$, consisting of a sequence of Internal nodes with a Leaf node or an Empty node at the end. We say a subpath of path $\left(\right.$ keys $\left.^{k}\right)$ is an active_path if all nodes from the root to node $n \in \operatorname{path}\left(k_{e y s}{ }^{k}\right)$ are active. Hence a physical_path $\left(k_{e y s}{ }^{k}\right)$ is active only if the end node is not moved.

Lemma 10. Two layers of dummy nodes are never changed.

Lemma 11. Children of a node with a Compress op will not be changed.

Lemma 12. Only an Internal node with all children Empty could be attached with a Compress op.

Lemma 13. An Internal node whose op is not Compress is active. 
Lemma 14. After the invocation of find(keys) which reads $l^{k}$, there is a snapshot in which the path from the root to $l^{k}$ is physical_path $\left(\right.$ keys $\left.^{k}\right)$.

Lemma 15. After ireplace, rreplace, mreplace, and creplace, a quadtree's properties remain.

Proof. We shall prove that in any snapshot $T_{i}$, a quadtree's properties remain.

First, Lemma 10 shows that two layers of dummy nodes remain in the tree. We have to consider other layers of nodes that are changed by replace operations.

Consider creplace that replaces an Internal node by an Empty node. Because the Internal node has been flagged on a Compress op before creplace (Lemma 8), all of its children are Empty and not changed (Lemma 11 and Lemma 12). Thus, creplace does not affect the second claim of Definition 1.

Consider ireplace, rreplace, or mreplace that replaces a terminal node by an Empty node, a Leaf node, or a subtree. By Lemma 7, before replace ${ }^{k}$, op.parent ${ }^{k}$ is flagged with $o p$ such that no successful replace operation could happen on op.parent ${ }^{k}$. Therefore, if the new node is Empty, it does not affect the tree property. If the new node is a Leaf node or a sub-tree, based on the post-conditions of the createNode function (Lemma 3), after replace operations the second claim of Definition 1 still holds.

Claim 2. A quadtree maintains its properties in every snapshot.

\subsection{Linearizability}

In this section, we define linearization points for basic operations. As the compress function is included in the move operation and the remove operation that return true, it does not affect the linearization points of these operations. If an algorithm is linearizable, its result be ordered equivalently as a sequential history by the linearization points. Since all modifications depend on find(keys), we first point out its linearization point. For find(keys), we define its linearization point at $T_{i}$ such that $l^{k}$ returned is on the physical_path $\left(\right.$ keys $\left.^{k}\right)$ in snapshot ${ }_{T_{i}}$.

For the contain operation that returns true, we show that there is a corresponding snapshot in which $l^{k}$ in physical_path $\left(k_{e y s}{ }^{k}\right)$ is active. For the contain operation, the insert operation, the remove operation, or the move operation that returns false, we show there is a corresponding snapshot in which $l^{k}$ in physical_path $\left(k e y s^{k}\right)$ is inactive. For the insert, remove, and move operations that returns true, we define the linearization point to be its first successful replace operation-replace ${ }_{i}^{0}$. To make a reasonable demonstration, we first show that replace ${ }_{i}^{k}, 0 \leq$ $k<\mid$ replace $_{i} \mid$ belongs to each operation that creates $o p_{i}$ and illustrate that $o p$ is unique for each operation.

Lemma 16. For find(keys) that returns tuples $\left\langle l^{k}, p O p^{k}\right.$, path $\left.^{k}\right\rangle$, there is a snapshot $T_{i}$ such that path ${ }^{k}$ returned with $l^{k}$ at the end is physical_path $\left(k e y^{k}\right)$ in snapshot $T_{i}$.

Lemma 17. If the insert operation, the remove operation, or the move operation returns true, the first successful replace operation occurs before returning, and it belongs to the op created by the operation itself at the last iteration in the while loop.
Lemma 18. If the insert operation, the remove operation, or the move operation returns false, there is no successful replace happens during the execution.

The next lemma points out the linearization points of the contain operation.

Lemma 19. For the contain operation that returns true, there is a corresponding snapshot that $l^{k}$ in physical_path $\left(k e y s^{k}\right)$ is active in snapshot $T_{i}$. For the contain operation that returns false, there is a corresponding snapshot that $l^{k}$ in physical_path $\left(\right.$ keys $\left.^{k}\right)$ is inactive in snapshot $T_{T_{i}}$.

We list out the linearization points of other operations as follows:

- insert(key). The linearization point of the insert operation that returns false is $T_{i}$ after calling find(key) at which $l$ at the end of physical_path(key) contains the key and is not moved. For the insert operation that returns true, the linearization point is at the first successful replace operation (line 145).

- remove(key). The linearization point of the remove operation that returns false is $T_{i}$ after calling find(key) at which $l$ at the end of physical_path(key) does not contain the key or is moved. For the successful remove operation, we define the linearization point where the node with key is replaced by an Empty node (line 145).

- move(oldKey, newKey). For the unsuccessful move operation, the linearization point depends on both newKey and oldKey. If $r l$ at the end of physical_path(oldKey) does not contain oldKey or is moved, the linearization point is $T_{i 1}$ after calling find(keys). Or else, if $i l$ at the end of physical_path(newKey) contains newKey and is not moved, the linearization point is at $T_{i 2}$ after calling find(keys).

For the successful move operation, the linearization point is the first successful replace operation. (line 255 or line 257)

Claim 3. quadboost is linearizable.

\subsection{Non-blocking}

Finally, we prove that quadboost is non-blocking, which means that the system as a whole is making progress even if some threads are starving.

Lemma 20. A node with a Compress op will not be pushed into path more than once.

Lemma 21. For path $\left(\right.$ keys $\left.^{k}\right)$, if $n_{t}$ is active in snapshot $T_{T_{i}}$, then $n_{0}, \ldots, n_{t-1}$ pushed before $n_{t}$ are active.

Lemma 22. If $n$ is the LCA node in snapshot $_{T_{i}}$ on physical_path for oldKey and newKey. Then at $T_{i 1}, T_{i 1}>$ $T_{i}, n$ is still the LCA on active_path for oldKey and newKey if it is active.

Lemma 23. path ${ }^{k}$ returned by find(keys) consists of finite number of keys.

Lemma 24. There is a unique spatial order among nodes in a quadtree in every snapshot. 
Lemma 25. There are a finite number of successful flag $\rightarrow$ replace $\rightarrow$, flag $\rightarrow$ replace, flag $\rightarrow$ unflag transitions.

Lemma 26. If the help function returned at $T_{i}$, and find(keys) at the prior iteration reads $p^{k}$.op at $T_{i 1}<T_{i}$, keys in snapshot $T_{T_{i}}$ and snapshot $T_{i 1}$ are different.

Claim 4. quadboost is non-blocking.

Proof. We have to prove that no process will execute loops infinitely without changing keys in a quadtree. First, we prove that path is terminable. Next, we prove that $\operatorname{find}\left(\right.$ keys $\left.^{k}\right)$ starts from an active node in physical_path $\left(\right.$ keys $\left.^{k}\right)$ in snapshot $T_{T_{i}}$ between $i_{t h}$ iteration and $i+1_{t h}$ iteration. Finally, some Leaf nodes in snapshot $_{T_{i 1}}$ at the returning of $\operatorname{find}($ keys $)$ at $i_{\text {th }}$ iteration are different from snapshot $T_{i 2}$ at the returning of find(keys) at $i+1_{t h}$ iteration.

For the first part, we initially start from the root node. Therefore, path is empty. Moreover, as Lemma 22 shows that $p a t h^{k}$ consists of finite number of keys, we establish this part.

For the second part, the continueFind function and the continueFindCommon function pop all nodes with Compress $o p$ from path. For the insert operation and remove operation, since Lemma 13 shows that an Internal nodes whose op is not Compress is active, and Lemma 21 shows that nodes above the active node are also active, there is a snapshot in which the top node of path is still in physical_path $\left(\right.$ keys $\left.^{k}\right)$. For the move operation, if either $r$ Fail or $i F a i l$ is true, it is equivalent with the prior case. If $c F a i l$ is true, Lemma 22 illustrates that if the LCA node is active, it is in physical_path for both oldKey and newKey. Thus, there is also a snapshot that the start node is in physical_path.

The third part is proved by contradiction, assuming that a quadtree is stabilized at $T_{i}$, and all invocations after $T_{i}$ are looping infinitely without changing Leaf nodes.

For the insert operation and the remove operation, before the invocation of $f$ ind(keys) at the next iteration, they must execute the help function at line 118 and line 175 accordingly. In both cases, the help function changes the snapshot (Lemma 26).

For the move operation, different situations of the continueFindCommon function are considered. If $r F$ ail or $i$ Fail is true, two situations arise: (1) $i O p$ or $r O p$ is Clean but $m$ flag fails; or (2) $i O p$ or $r O p$ is not Clean. In both cases, before the invocation of $\operatorname{find}\left(\mathrm{keys}^{k}\right)$, the help function is performed at line 311 and line 287 Thus by Lemma 26, the snapshot is changed between two iterations. Now consider if $c F$ ail is true. If $i p$ and $r p$ are the same, it could result from the difference between $r O p$ and $i O p$. In this case, the snapshot might be changed between reading $r O p$ and $i O p$. It could also result from the failure of mflag. For the above cases, the help function at line 332 would change the quadtree. If $i p$ and $r p$ are different, it results from either $i$ Path or rPath that have popped the LCA node. We have proved the case in which either $i F a i l$ or $r F a i l$ is true and derives a contradiction.

From the above discussions, we prove that quadboost is non-blocking.

\section{Evaluation}

We ran experiments on a machine with 64GB main memory and two 2.6GHZ Intel(R) Xeon(R) 8-core E5-2670 processors with hyper-threading enabled, rendering 32 hardware threads in total. We used the RedHat Enterprise Server 6.3 with Linux core 2.6.32, and all experiments were ran under Sun Java SE Runtime Environment (build 1.8.0_65). To avoid significant run-time garbage collection cost, we set the initial heap size to 6GB.

For each experiment, we ran eight 1-second cases, where the first 3 cases were performed to warm up JVM, and the median of the last 5 cases was used as the real performance. Before the start of each case, we inserted half keys from the key set into a quadtree to guarantee that the insert and the remove operation have equal success opportunity initially.

We apply uniformly distributed key sets that contain two-dimensional points within a square. We use the range to denote the border of a square. Thus, points are located inside a range $*$ range square. In our experiments, we used two different key sets: $10^{2}$ keys to measure the performance under high contention, and $10^{6}$ keys to measure the performance under low contention. For simplicity, we let the range of the first category experiment be 10 , rendering $1-10^{2}$ consecutive keys for one-dimensional structure. For the second category experiment, we let the range be 1000 , generating $1-10^{6}$ consecutive keys.

TABLE 1: The concurrent quadtree algorithms with different optimization strategies.

\begin{tabular}{|c|c|c|c|}
\hline type & insert & remove & move \\
\hline $\mathbf{q c}$ & single CAS & single CAS & not support \\
\hline qb-s & $\begin{array}{c}\text { flag CAS, } \\
\text { continuous find, } \\
\text { stack }\end{array}$ & $\begin{array}{c}\text { continuous find, } \\
\text { decoupling stack, } \\
\text { recursive compression }\end{array}$ & $\begin{array}{c}\text { continuous find, } \\
\text { decoupling stack, } \\
\text { recursive compression }\end{array}$ \\
\hline $\mathbf{q b - o}$ & $\begin{array}{c}\text { flag CAS, } \\
\text { continuous find }\end{array}$ & $\begin{array}{c}\text { flag CAS, } \\
\text { continuous find, } \\
\text { decoupling compression }\end{array}$ & $\begin{array}{c}\text { flag CAS, } \\
\text { continuous find, } \\
\text { decoupling compression }\end{array}$ \\
\hline
\end{tabular}

We evaluate quadboost algorithms via comparing with the state-of-the-art concurrent trees (kary, ctrie, and patricia) for throughput (Section 6.1) and presenting the incremental effects of the optimization strategies proposed in this work (Section 6.2). Table 1 lists the concurrent quadtree algorithms. qb-o (quadboost-one parent) is the one parent optimization based on qb-s (quadboost-stack) mentioned in Section 4.4. qc is the CAS quadtree introduced in Section 3.

\subsection{Throughput}

To the best of our knowledge, a formal concurrent quadtree has not been published yet. Hence, we compare our quadtrees with three one-dimensional non-blocking trees:

- kary is a non-blocking k-way search tree, where $k$ represents the number of branches maintained by an internal node. Like the non-blocking BST [4], keys are kept in leaf nodes. When $k=2$, the structure is similar as the non-blocking BST; when $k=4$, each internal node has four children, it has a similar structure to quadtree. However, kary's structure depends on the modification order, and it does not have a series of internal nodes representing the twodimensional space hierarchy.

- ctrie is a concurrent hash trie, where each node can store up to $2^{k}$ children. We use $k=2$ to make a 
4-way hash trie that resembles quadtree. The hash trie also incorporates a compression mechanism to reduce unnecessary nodes. Different from quadtree, it uses a control node (INODE as the paper indicates) to coordinate concurrent updates. Hence, the search depth could be longer than quadtree.

- patricia is a binary search tree, which adopts Ellen's BST techniques [4]. As the author points out, it can be used as quadtree by interleaving the bits of $x$ and $y$. It also supports the move (replace) operation like quadboost. Unlike our LCA-based operation, it searches two positions separately without a continuous find mechanism.

Since the above structures only store one-dimensional keys, we had to transform a two-dimensional key into a one-dimensional key for comparison. Though patricia could store two-dimensional keys using the above method, ctrie and kary cannot use it. Thus, we devised a general formula: $k e y^{1}=k e y_{x}^{2} *$ range $+k e y_{y}^{2}$. Given a two-dimensional key$k e y^{2}$, and range, we transformed it into a one-dimensional key-key ${ }^{1}$. To refrain from trivial transformations by floating numbers, we only considered integer numbers in this section.

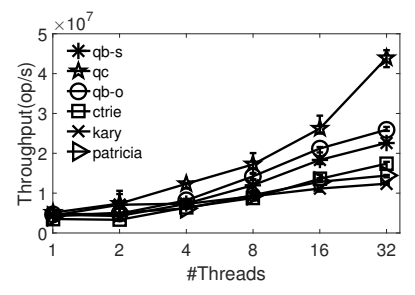

(a) $10^{2}$ keys

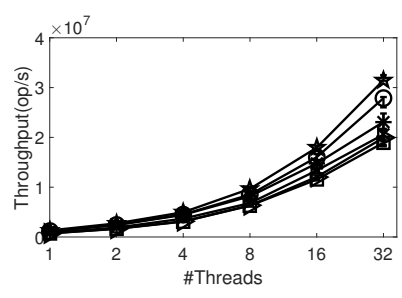

(b) $10^{6}$ keys
Fig. 15: Throughput of different concurrent trees under both high and low contention (50\% insert, 50\% remove).

Due to the lack of the move operation in CAS quadtree (qc), we compare throughput with/without the move operation respectively. Figure 15 plots throughput without any move operation for the concurrent algorithms. It is not surprising to observe that qc achieves the highest throughput. To some extent, qc represents an upper bound of throughput because it maintains the hierarchy without physical removal, i.e., its remove operation only applies a CAS on edges to change links, which leads to less contention than other practical concurrent algorithms. However, both qb-s and qb-o can achieve comparable throughput when the key set becomes larger. This phenomenon occurs due to: (i) given the large key set, fewer thread interventions results in a lesser number of CAS failures on nodes; and (ii) both algorithms compress nodes and use the continuous find mechanism to reduce the length of traverse path.

As a comparison, ctrie, kary, and patricia show lower performances with the increasing number of threads. For instance, in Figure $15 a$ at 32 threads, qb-o outperforms ctrie by $49 \%$, patricia by $79 \%$, and kary by $109 \%$. Note that qb$\mathbf{o}$ and $\mathbf{q b}$-s incorporate the continuous find mechanism to reduce the length of traverse path. Further, both kary and patricia flag the grandparent node in the remove operation, which allows less concurrency than ctrie, qb-o and $\mathbf{q b}$ - s with the decoupling approach shown in Figure 7 , qb$\mathbf{s}$ is worse than $\mathbf{q b - o}$ due to its extra cost of recording elements and compressing nodes recursively. Figure 15b exhibits results from when the key set was large. There is less collision among threads but deeper depths of trees than the smaller key set. In the scenario, $\mathbf{q b}$-o and $\mathbf{q b}$-s show a similar performance as qc because of less number of CAS failures caused by thread interventions. qb-o is only $12 \%$ worse than qc at 32 threads, but $47 \%$ better than ctrie, $35 \%$ better than kary, and 39\% better than patricia mainly for its shorter traversal paths caused by its static representation and the continuous find mechanism. As we discussed in the next section, $\mathbf{q b}$-s and $\mathbf{q b}$-o save a significant number of nodes as shown in Figure 18. It implies that $\mathbf{q b}$-s and qb-o occupy less memory and result in a shorter path for traversal.

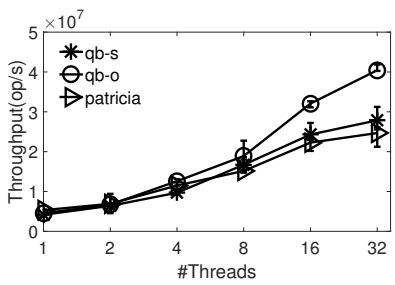

(a) $10^{2}$ keys

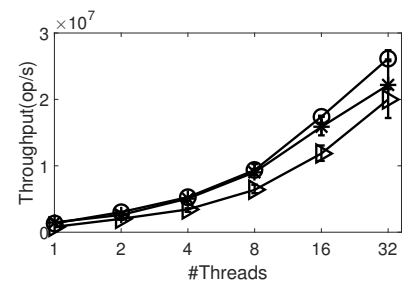

(b) $10^{6}$ keys
Fig. 16: Comparison of the move operation's throughput between quadboost and patricia in both small and large ranges (10\% insert, $10 \%$ remove, $80 \%$ move).

Figure $16 b$ demonstrates that quadboost has an efficient move operation. Using the small key set, where the depth is not a significant impact, Figure 16a shows that qb-o is more efficient than patricia especially when contention is high. For example, it performs better than patricia by $47 \%$ at 32 threads because it adopts the continuous find mechanism to traverse less path and decouples physical adjustment for higher concurrency. However, qb-s is similar to patricia since it has to maintain a stack and recursively compress nodes in a quadtree. Figure $16 \mathrm{~b}$ illustrates that qb-s and qb-o have a similar throughput for the large key set. qb-o outperforms patricia by $31 \%$ at 32 threads. When the key set is large, the depth becomes a more significant factor due to less contention. Since each Internal node in a quadtree maintains four children while patricia maintains two, the depth of patricia is deeper than quadboost. Further, the combination of the LCA node and the continuous find mechanism ensures that $\mathbf{q b}-\mathbf{o}$ and $\mathbf{q b}$-s do not need to restart from the root even if flags on two different nodes fail.

\subsection{Analysis}

To determine how quadboost algorithms improve the performance, we devised two algorithms that incrementally use parts of techniques in qb-o:

- $\mathbf{q b}$-f flags the parent of a terminal node in the move operation, the insert operation, and the remove operation. It restarts from the root without a continuous find mechanism. Further, it adopts the traditional remove mechanism mentioned in Figure $7 \mathrm{a}$ 
- qb-d decouples the physical adjustment in the remove operation based on qb-f.

range here was set to $2^{32}-1$, and both $k e y_{x}$ and $k e y_{y}$ could be floating numbers. We used an insert dominated experiment and a remove dominated experiment to demonstrate the effects of different techniques. We used a remove dominated experiment to show the effect of decoupling, where insert:remove ratio was 1:9. In the insert dominated experiment, the insert:remove ratio was 9:1; hence, there were far more insert operations. Since fewer compress operations were induced, the experiment showed the effect of the continuous find mechanism. Figure $17 \mathrm{a}$ illustrates

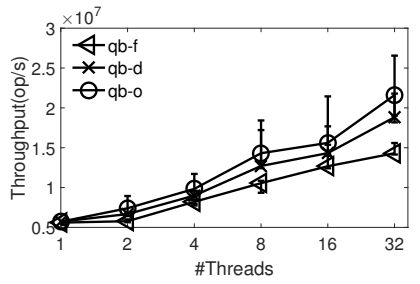

(a) 10\% insert, 90\% remove

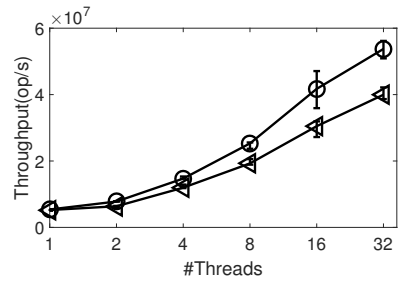

(b) $90 \%$ insert, $10 \%$ remove
Fig. 17: Throughput comparison in insert dominated and remove dominated cases $\left(10^{2}\right.$ keys).

that quadtrees with decoupling exhibit a higher throughput than $\mathbf{q b}-\mathbf{f}$, the basic flag concurrent quadtree. Besides, qbo which incorporates the continuous find is more efficient than $\mathbf{q b}$-d. Specifically, at 32 threads, qb-o performs $15 \%$ better than $\mathbf{q b}$-d and $51 \%$ better than $\mathbf{q b}$-f. From Figure $17 \mathrm{~b}$ we determine that $\mathbf{q b}$-o outperforms $\mathbf{q b}$-f by up to $35 \%$. Therefore, it demonstrates that the continuous find mechanism and the decoupling approach play a significant role in our algorithm.

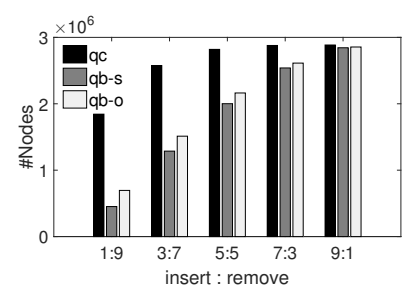

(a) Nodes

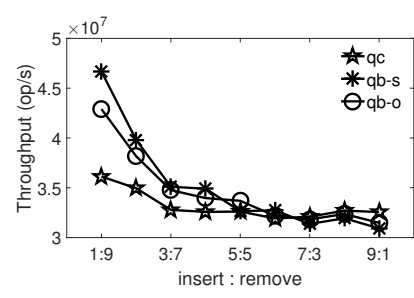

(b) Throughput
Fig. 18: Number of nodes left and throughput under different ratios of insert:remove from 9:1 to 1:9, with fixed 90\% contain, under 32 threads and $10^{6}$ keys.

Another advantage of quadboost results from the compression technique, which reduces the search path for each operation and the memory consumption. Figure 19 plots the number of nodes left and the throughput of each quadtree at different insert:remove ratios. As qc only replaces a terminal node with an Empty node without compression, it results in the greatest number of nodes in the memory (Figure 18a). In contrast, $\mathbf{q b}$-s and $\mathbf{q b} \mathbf{b}-\mathbf{o}$ compress a quadtree if necessary. When qc contains much more nodes than other quadtrees, the remove operation dominates (the first groups

2. Unlike previous experiments, we run eight 3-second cases in the experiment to ensure stable amount of modifications bars to the left) and has three times the amount of nodes of $\mathbf{q b}$-s. The result also indicates that $\mathbf{q b}$-o contains a similar number of nodes to $\mathbf{q b} \mathbf{b}$-s despite it only compresses one layer of nodes. Figure $18 \mathrm{~b}$ illustrates the effectiveness of compression in the face of tremendous contain operations. qb-s outperforms qc by 30\% at 9:1 insert:remove ratio because qb-s adjusts the quadtree's structure by compression to reduce the length of the search path. With the increment of the insert ratio, $\mathbf{q b}$-s performs similar to qc due to the extra cost of maintaining a stack and the recursive compression. However, qb-o achieves good balance between $\mathbf{q b}$-s and $\mathbf{q c}$, which compresses one layer of nodes without recording the whole traverse path.

\section{Related Works}

Because there are few formal works related to concurrent quadtrees, we present a roadmap to show the development of state-of-the-art concurrent trees in Figure 19.

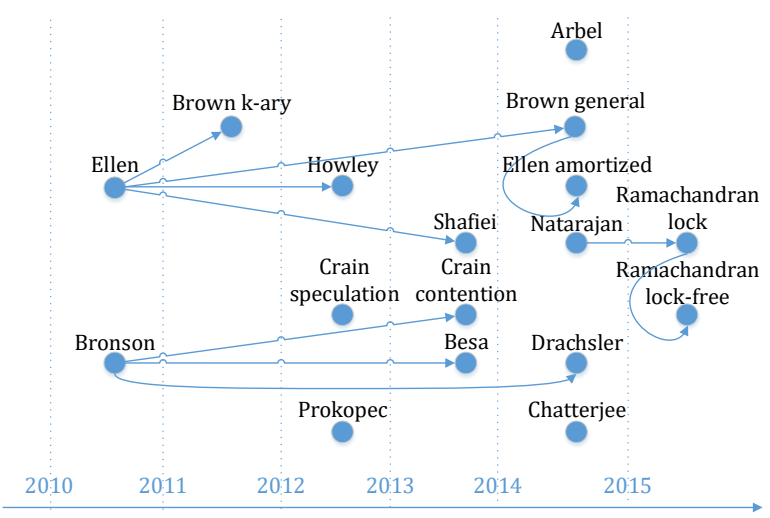

Fig. 19: Concurrent trees roadmap

Ellen [4] provided the first non-blocking BST and proved it correct. This work is based on the cooperative method described in Turek [17] and Barnes [18]. Brown [19] used a similar approach for the concurrent k-ary tree. Shafiei [20] also applied the method for the concurrent patricia trie. It also showed how to design a concurrent operation where two pointers need to be changed. The above concurrent trees have an external structure, where only leaf nodes contain actual keys. Howley [5] designed the first internal BST by the cooperative method. Recently, Brown [21] presented a generalized template for all concurrent down-trees. Ellen [22] exhibited how to incorporate a stack to reduce the original complexity from $O(c h)$ to $O(h+c)$. Our quadboost is a hybrid of these techniques. It uses a cooperative method for concurrent coordination, changes two different positions with atomicity, and devises a continuous find mechanism to reduce restart cost.

Different from the previously mentioned methods that apply flags on nodes, Natarajan [6] illustrated how to apply flags on edges for a non-blocking external BST. Ramachandran $|7|$ adopted CAS locks on edges to design a concurrent internal BST and later extended the work to nonblocking [8]. Their experiments showed that internal trees are more scalable than external trees with a large key range. On one hand, internal trees take up less memory; on the other hand, the remove operation is more complicated than 
that in external trees. Chatterjee [9] provided a threadedBST with edge flags and claimed it has a low theoretical complexity. Unlike the above trees that have to flag their edges before removal, our CAS quadtree uses a single CAS in both the insert operation and the remove operation.

The first balanced concurrent BST was proposed by Bronson [23] in which they used an optimistic and relaxed balance method to build an AVL tree. Besa |24| employed a similar method for a red-black tree. Crain [25] proposed a method that decouples physical adjustment from logical removal by a background thread. Drachsler [10] mentioned an alternative technique called logical ordering, which uses the key order of the BST to optimize the contain operation. All of these works were constructed on fine-grained locks and are deadlock free. Based on the special properties of quadtree, we also decoupled physical adjustment from logical removal and achieved a high throughput.

There are numerous studies on concurrent trees in addition to ours and the ones mentioned in this work. Crain [26] designed a concurrent AVL tree based on STM. Prokopec [27] used a control node, which is similar to our Operation object to develop a concurrent trie. Arbel [28] provided a balanced BST with RCU and fine-grained locks.

\section{CONCLUSIONS}

In this paper, we present a set of concurrent quadtree algorithms-quadboost, which support concurrent insert, remove, contain, and move operations. In the remove operation, we decouple physical updates from the logical removal to improve concurrency. The continuous find mechanism analyses flags on nodes to decide whether to move down or up. Further, our LCA-based move operation can modify two pointers with atomicity. The experimental results demonstrate that quadboost outperforms existing one-dimensional tree structures while maintaining a two-dimensional hierarchy. The quadboost algorithms are scalable with a variety of workloads and thread counts.

\section{REFERENCES}

[1] M. Moir and N. Shavit, "Concurrent data structures," Handbook of Data Structures and Applications, pp. 47-14, 2007.

[2] N. Shavit, "Data structures in the multicore age," Communications of the ACM, vol. 54, no. 3, pp. 76-84, 2011.

[3] T. David, R. Guerraoui, and V. Trigonakis, "Asynchronized concurrency: The secret to scaling concurrent search data structures," in Proceedings of the Twentieth International Conference on Architectural Support for Programming Languages and Operating Systems. ACM, 2015, pp. 631-644.

[4] F. Ellen, P. Fatourou, E. Ruppert, and F. van Breugel, "Nonblocking binary search trees," in Proceedings of the 29th ACM SIGACT-SIGOPS symposium on Principles of distributed computing. ACM, 2010, pp. 131-140.

[5] S. V. Howley and J. Jones, "A non-blocking internal binary search tree," in Proceedings of the twenty-fourth annual ACM symposium on Parallelism in algorithms and architectures. ACM, 2012, pp. 161-171.

[6] A. Natarajan and N. Mittal, "Fast concurrent lock-free binary search trees," in ACM SIGPLAN Notices, vol. 49, no. 8. ACM, 2014, pp. 317-328.

[7] A. Ramachandran and N. Mittal, "Castle: fast concurrent internal binary search tree using edge-based locking," in Proceedings of the 20th ACM SIGPLAN Symposium on Principles and Practice of Parallel Programming. ACM, 2015, pp. 281-282.

[8] - "A fast lock-free internal binary search tree," in Proceedings of the 2015 International Conference on Distributed Computing and Networking. ACM, 2015, p. 37.
[9] B. Chatterjee, N. Nguyen, and P. Tsigas, "Efficient lock-free binary search trees," in Proceedings of the 2014 ACM symposium on Principles of distributed computing. ACM, 2014, pp. 322-331.

[10] D. Drachsler, M. Vechev, and E. Yahav, "Practical concurrent binary search trees via logical ordering," in ACM SIGPLAN Notices, vol. 49, no. 8. ACM, 2014, pp. 343-356.

[11] V. Ng and T. Kameda, "Concurrent accesses to r-trees," in Advances in Spatial Databases. Springer, 1993, pp. 142-161.

[12] J. Chen, Y.-F. Huang, and Y.-H. Chin, "A study of concurrent operations on r-trees," Information Sciences, vol. 98, no. 1, pp. 263300, 1997.

[13] R. Obe and L. Hsu, PostGIS in action. Manning Publications Co., 2011.

[14] A. C. Tassio Knop, “Qollide - Quadtrees and Collisions," https:// graphics.ethz.ch/ achapiro/gc.html. 2010, [Online; accessed 29August-2015].

[15] G. J. Sullivan and R. L. Baker, "Efficient quadtree coding of images and video," Image Processing, IEEE Transactions on, vol. 3, no. 3, pp. 327-331, 1994

[16] D. P. Mehta and S. Sahni, Handbook of data structures and applications. CRC Press, 2004.

[17] J. Turek, D. Shasha, and S. Prakash, "Locking without blocking: making lock based concurrent data structure algorithms nonblocking," in Proceedings of the eleventh ACM SIGACT-SIGMOD-SIGART symposium on Principles of database systems. ACM, 1992, pp. 212 222.

[18] G. Barnes, "A method for implementing lock-free shared-data structures," in Proceedings of the fifth annual ACM symposium on Parallel algorithms and architectures. ACM, 1993, pp. 261-270.

[19] T. Brown and J. Helga, "Non-blocking k-ary search trees," in Principles of Distributed Systems. Springer, 2011, pp. 207-221.

[20] N. Shafiei, "Non-blocking patricia tries with replace operations," in Distributed Computing Systems (ICDCS), 2013 IEEE 33rd International Conference on. IEEE, 2013, pp. 216-225.

[21] T. Brown, F. Ellen, and E. Ruppert, "A general technique for nonblocking trees," in ACM SIGPLAN Notices, vol. 49, no. 8. ACM, 2014, pp. 329-342.

[22] F. Ellen, P. Fatourou, J. Helga, and E. Ruppert, "The amortized complexity of non-blocking binary search trees," in Proceedings of the 2014 ACM symposium on Principles of distributed computing. ACM, 2014, pp. 332-340.

[23] N. G. Bronson, J. Casper, H. Chafi, and K. Olukotun, "A practical concurrent binary search tree," in ACM Sigplan Notices, vol. 45, no. 5. ACM, 2010, pp. 257-268.

[24] J. Besa and Y. Eterovic, "A concurrent red-black tree," Journal of Parallel and Distributed Computing, vol. 73, no. 4, pp. 434-449, 2013.

[25] T. Crain, V. Gramoli, and M. Raynal, "A contention-friendly binary search tree," in Euro-Par 2013 Parallel Processing. S Springer, 2013, pp. 229-240.

[26] $\frac{\mathrm{T}}{\mathrm{N}}$, "A speculation-friendly binary search tree," Acm Sigplan Notices, vol. 47, no. 8, pp. 161-170, 2012.

[27] A. Prokopec, N. G. Bronson, P. Bagwell, and M. Odersky, "Concurrent tries with efficient non-blocking snapshots," in Acm Sigplan Notices, vol. 47, no. 8. ACM, 2012, pp. 151-160.

[28] M. Arbel and H. Attiya, "Concurrent updates with rcu: Search tree as an example," in Proceedings of the 2014 ACM symposium on Principles of distributed computing. ACM, 2014, pp. 196-205. 


\section{APPENDIX}

\section{.1 Basic Invariants}

We provide a detailed proof of quadboost in this section. We follow the same naming convention in the paper. There are four kinds of basic operations in quadboost, i.e. the insert operation, the remove operation, the move operation, and the contain operation. Other functions are called subroutines, which are invoked by basic operations. The insert operation and the remove operation only operate on one terminal node, whereas the the move operation operates two different terminals-one for inserting a node with newKey, the other is for removing a node with oldKey. We call them newKey's terminal and oldKey's terminal, and we call their parents newKey's parent and oldKey's parent accordingly. Moreover, we name a CAS that changes a node's op a flag operation and a CAS that changes a node's child a replace operation. We define snapshot $T_{i}$ as the state of our quadtree at some time $T_{i}$.

\section{.1.1 Subroutines}

To begin with, we have following observations from quadboost.

Observation 1. The key field of a Leaf node is never changed. The op field of a Leaf node is initially null.

Observation 2. The space information- $\langle x, y, w, h\rangle$ of an Internal node is never changed.

Observation 3. The root node is never changed.

Based on Observation 3, we derive a Corollary as follows:

Corollary 1. Two nodes in quadtree must share a common search path starting from the root.

Using these observations, we prove that each subroutine satisfies their specific pre-conditions and postconditions. Because all basic operations invoke the find function at line 134 that returns $\langle l, p O p, p a t h\rangle$ and the findCommon function at line 270 that returns $\langle i l, i O p, i P a t h, r l, r O p, r P a t h\rangle$, we prove that the two functions satisfy their pre-conditions and post-conditions beforehand. We suppose that they execute from a snapshot $_{T_{i}}$ to derive these conditions.

Definition 1. The pre-condition of find (l, pOp, path, keyX, keyY) invoked at $T_{i}$ :

1) If path is not empty, $l$ was on the direction $d \in$ $\{n w, n e, s w, s e\}$ of the top node in path at $T_{i 1} \leq T_{i}$.

The post-conditions of find(l, pOp, path, keyX, keyY) that returns a tuple $\langle l, p O p$, path $\rangle$ at $T_{i}$ :

1) $l$ is a Leaf node or an Empty node.

2) At some $T_{i 1}<T_{i}$, the top node in path has contained $p O p$.

3) At some $T_{i 1}<T_{i}$, the top node in path has contained $l$.

4) If $p O p$ was read at $T_{i 1}$, and $l$ was read at $T_{i 2}$, then $T_{i 1}<T_{i 2}<T_{i}$.

5) For each node $n$ in path, size (path) $\geq 2, n_{t}$ is on the top of $n_{t-1}$, and $n_{t}$ was on the direction $d \in$ $\{n w, n e, s w, s e\}$ of $n_{t-1}$ at $T_{i 1} l e q T_{i}$.
Definition 2. The pre-conditions of findCommon(il, $r l, l c a, r O p$, iOp, iPath, rPath, oldKeyX, oldKeyY, newKeyX, newKeyY) invoked at $T_{i}$ :

1) If iPath is not empty, il was on the direction $d \in$ $\{n w, n e, s w, s e\}$ of the top node in iPath at $T_{i 1} \leq T_{i}$.

2) If $r$ Path is not empty, $r l$ was on the direction $d \in$ $\{n w, n e, s w, s e\}$ of the top node in $r$ Path at $T_{i 1} \leq T_{i}$.

The post-conditions of findCommon(il, rl, lca, rOp, iOp, iPath, rPath, oldKeyX, oldKeyY, newKeyX, newKeyY) that returns two tuples $\langle i l, i O p, i P a t h\rangle$ and $\langle r l, r O p, r P a t h\rangle$ at $T_{i}$ :

1) $r l$ was a Leaf or an Empty node.

2) il was a Leaf or an Empty node.

3) At some $T_{i 1}<T_{i}$, the top node in $r$ Path has contained $r O p$.

4) At some $T_{i 1}<T_{i}$, the top node in iPath has contained $i O p$.

5) At some $T_{i 1}<T_{i}$, the top node in $r$ Path has contained $r l$.

6) At some $T_{i 1}<T_{i}$, the top node in iPath has contained il.

7) If $i O p$ was read at $T_{i 1}$, and $i l$ was read at $T_{i 2}$, then $T_{i 1}<T_{i 2}<T_{i}$.

8) If $r O p$ was read at $T_{i 1}$, and $r l$ was read at $T_{i 2}$, then $T_{i 1}<T_{i 2}<T_{i}$.

9) For each node $n$ in $r$ Path, size (rPath) $\geq 2, n_{t}$ is on the top of $n_{t-1}$, and $n_{t}$ was on the direction $d \in$ $\{n w, n e, s w, s e\}$ of $n_{t-1}$ at $T_{i 1} \leq T_{i}$.

10) For each node $n$ in iPath, size (iPath) $\geq 2, n_{t}$ is on the top of $n_{t-1}$, and $n_{t}$ was on the direction $d \in$ $\{n w, n e, s w, s e\}$ of $n_{t-1}$ at $T_{i 1} \leq T_{i}$.

By observing the find function and the findCommon function, we have following lemmas.

Lemma 1. At the first time calling the find function, the contain operation, the insert operation, and the remove function start with $l$ as an Internal node and an empty path.

Proof. The contain operation at line 97 , the insert operation at line 105 and the remove operation at line 161 start with the root node by $l=$ root, an Internal node that is never changed (Observation 3 ).

Besides, the contain operation at line 95 , the insert operation at line 103 , and the remove operation at line 160 start with an empty stack to record nodes.

Lemma 2. At the first time the move operation calls the find Common function, it starts with $r l$ as an Internal node and $r$ Path and $i$ Path are empty.

Proof. The move operation begins with the root node at line by $r l=$ root that is never changed (Observation 3 ) at line 204 Both $r$ Path and $i P a t h$ are initialized as empty stacks at line 199 .

Lemma 3. All nodes pushed by the find function (line 134) and the continueFind function (line 122) are Internal nodes.

Proof. Before pushing into the stack, the find function at line 135 first check the class of a node. Also, the continueFind function calls the find function at line 132 to push nodes into path. Thus, nodes other than Internal cannot be pushed. 
Lemma 4. All nodes pushed by the findCommon function (line 270) the continueFindCommon function (line 285) are Internal.

Proof. Before pushing into the stack, the findCommon function first checks the class of a node at line 271 Also, the continueFindCommon function calls the findCommon function at line 342 . Or it calls the find function at line 323 and line 302 to push Internal nodes according to Lemma 3 Thus, nodes other than Internal cannot be pushed.

Lemma 5. For the loop in the find function at line 134 and the findCommon function at line 270. we suppose that path ( $r$ Path) is empty and refer $l(r l)$ and $p O p(r O p)$ to each field updated by the loop. If the loop executes at least once and breaks at $T_{i}, l(r l)$, $p O p(r O p)$, and path (rPath) satisfy following conditions:

1) $\quad l(r l)$ is a Leaf node or an Empty node.

2) At some $T_{i 1}<T_{i}$, the top node in path(rPath) has contained $p O p(r O p)$.

3) At some $T_{i 1}<T_{i}$, the top node in path(rPath) has contained $l(r l)$.

4) If $p O p(r O p)$ was read at $T_{i 1}$, and $l(r l)$ was read at $T_{i 2}$, then $T_{i 1}<T_{i 2}<T_{i}$.

5) For each node $n$ in path $\left(r\right.$ Path), size (path) $\geq 2, n_{t}$ is on the top of $n_{t-1}$, and $n_{t}$ was on the direction $d \in$ $\{n w, n e, s w, s e\}$ of $n_{t-1}$ at $T_{i 1} \leq T_{i}$.

Proof. Apart from the terminate condition that the findCommon function judges whether two directions are the same or not, it performs in the same pattern as the find function. Both functions first push the previous Internal node $l^{\prime}$ into $p a t h$, then get its $o p$, and read a child pointer $l$ finally.

The first condition always holds because by Lemma 4 and Lemma 3 Leaf nodes and Empty nodes will never be pushed into the path(rPath).

We then prove part 2-4. At the last iteration, $p O p(r O p)$ and $l(r l)$ are read from $l^{\prime}$, which has already been pushed into the path(rPath). Hence, part 2 and part 3 are correct. Besides, $p O p(r O p)$ is read before $l(r l)$ so that part 4 holds.

For the last part, we assume that at $T_{i 1}$ the lemma holds, so at $T_{i 2}>T_{i 1} l$ is read from $l^{\prime}$ which has already been pushed into the path(rPath). As path(rPath) is initially empty, and $T_{i}$ is at the end of the last iteration, $l$ which on the top of $l^{\prime}$ has been a child of it.

Lemma 5 proves loop within the find function and the findCommon function satisfy post-conditions in Definition 1 and Definition 2. Next parts are going to show that other statements do not change these properties. We consider the first invocation as the base case, and prove lemmas by induction.

Lemma 6. Every call to the find function satisfies its precondition and post-conditions.

Proof. Now we prove the base case. We prove that initially the lemma holds.

The pre-condition:

By Lemma 1. the contain operation, the insert operation, and the remove operation start with an empty stack to record nodes.

For the move operation, by Lemma 2 it starts with $r l$ as an Internal node and empty paths. Therefore it executes the loop more than once to push Internal nodes into path. In the last iteration $r l$ is pushed at line 272, and the parent of it has already been push at the prior iteration. Then, $r l$ is read from at line 278 Hence, $r l$ at line 279 is a child of the top node in $r P a t h$ if it is not empty. iPath is empty since it starts with an empty stack.

Therefore, initially the pre-conditions are satisfied.

The post-conditions:

The first condition always satisfies as the class of a node is checked at line 135

By Lemma 1. the contain operation, the insert operation, and the remove operation start with an empty stack. Thu Lemma 5 indicates that post-conditions are satisfied.

For the find function within the findCommon function, by Lemma 5 it satisfies post-conditions when it breaks out. Hence, at line 282, post-conditions are satisfied because iPath is empty. At line 279, if $r l$ starts as a Leaf node, the post-conditions are also satisfied. Otherwise, there are two parts of $r$ Path at the time the loop breaks out, where we have proved each of them satisfy post-conditions by Lemma 5 . Moreover, by the pre-condition, $r l$ has been a child of the top node in $r$ Path of the find function. Thus, all nodes pushed later also satisfy the post-conditions.

We have proved the base case. Then we assume that for invocations find $_{0}, f_{\text {ind }}$, find $_{2} \ldots$ find $_{k}$, the first $k$ invocations satisfy the pre-conditions and post-conditions. We should prove that find $_{k+1}$ also satisfy the conditions. We have to consider all places where the find function is invoked.

The pre-condition:

First we consider the contain operation, the insert operation, and the move operation.

At line 98, line 106, line 163, the invocations follow the base case which we have proved satisfy the pre-condition.

At line 132, there are two scenarios $l$ is read. $l$ could either be read from the path at line 126 or be assigned to its parent node at line 123 In the latter case, $p$ was at the top of path at $T_{i 1}<T_{i}$ (line 109 and line 166 by the hypothesis. Otherwise $l$ is assigned to a node in path. As there's no other push operations, $n_{t}$ on the top of $n_{t-1}$ must be its child at some $T_{i 1}<T_{i}$ by the hypothesis.

Then, we discuss the move operation.

At line 279, the find function is wrapped by the findCommon function. Since Lemma 5 shows that the loop set $r l$ as a child of the top node in rPath, we have to prove that either it is an Internal node or an Leaf node that was a child of the top node before entering the loop. At line 212 the findCommon function follows the base case. At line 342 . Corollary 1 shows that the root node will always be a LCA node. Hence, after setting $r P a t h$ to its lca index at line 334 . it contains at least one node. By Lemma 3 and Lemma $4 . r l$ is an Internal node popped from $r$ Path at line 337.

At line 282 the find function is also wrapped by the findCommon function. We could prove that $i$ Path is empty. At line 212, the findCommon function follows the base case. At line 342, iPath is set to empty before the invocation (line 335).

At line 302, $r l$ is either assigned to $r p$ (line 288 or a node popped from $r$ Path (line 295. If it is popped from $r$ Path, our hypothesis ensures that it was a child of the top node from $r$ Path. If it is read from $r p$, which is an Internal node 
popped from $r$ Path at line 214 line 344 , or line 305 , it also satisfies the claim.

At line 323. likewise, $i l$ is either assigned to $i p$ (line 312 or a node popped from $i$ Path (line 315). If it is popped from $i P a t h$, our hypothesis ensures that $i l$ was a child node of the top node from $i$ Path if it is not empty. If it is read from $i p$, which is an Internal node popped from iPath at line 213 line 345, or line 326, it also satisfies the claim.

The post-conditions:

The first condition always holds. Because by Lemma 3 if $l$ is an Internal node, it will be pushed into path.

We then prove other conditions.

First we consider the contain operation, the insert operation, and the move operation.

At line 98, line 106, line 163 , the invocations follow the base case which we have proved satisfy the post-conditions.

At line 132, there are two scenarios $l$ is read. $l$ could either be read from the path at line 126 or be assigned to its parent node at line 123 In both cases, $l$ was an Internal node in path. Therefore Lemma 5 indicates that all nodes followed by $l$ satisfy the post-conditions.

Then, we discuss the move operation.

At line 279 the find function is wrapped by the findCommon function. The pre-condition indicates $r l$ was a child of $r$ Path if it is not empty. Hence, it suffices to show that the post-conditions are satisfied by Lemma 5 .

At line 282, the find function is also wrapped by the findCommon function. The pre-condition indicates $i$ Path is an empty. In this way, by Lemma 5 we prove the postconditions.

At line 302, likewise, the pre-condition suggests that $r l$ was an Internal node in rPath. By Lemma 5 we also prove the post-conditions.

At line 323, the pre-condition suggests that $i l$ was an Internal node in iPath. Therefore, by Lemma 5 we prove the post-conditions.

After showing the pre-condition and post-conditions of the find function, we the prove that the findCommon function that returns $\langle i l, i O p, i P a t h, r l, r O p, r P a t h\rangle$ satisfies its pre-conditions and post-conditions.

Lemma 7. Every call to the findCommon function satisfies its pre-conditions and post-conditions.

Proof. The pre-condition:

We prove the lemma by induction. First we prove the base case.

At line 212. the findCommon function is initially invoked, and both $i$ Path and rPath are empty (line 199).

Then we assume that for invocations findCommon , $_{0}$ findCommon $_{1}$, findCommon $_{2}$... findCommon $k$, the first $k$ invocations satisfy the pre-conditions. We prove that findCommon $_{k+1}$ also satisfies the conditions.

There are two places the findCommon function is invoked. At line 212 the base case proves the post-conditions. At line 342. Corollary 1 shows that the root node will always be a LCA node. Hence, after setting $r$ Path to its lca index at line 334 it contains at least one node. By Lemma 3 and Lemma $4 r$ rl is an Internal node popped from $r P a t h$ at line 337 Hence, we prove the pre-condition.

The post-conditions:
The findCommon function wraps two find functions at line 282 and line 279 Because $\langle r l, r O p, r P a t h\rangle$ that passed from the find function for $r l$ and $\langle i l, i O p, i P a t h\rangle$ that passed from the find function for $i l$ hold the post-conditions, the lemma is true.

After proving the pre-conditions and post-conditions of the find function and the findCommon function, we shall prove that the continuous find mechanism (i.e. the continueFind function and the continueFindCommon function) satisfies its pre-conditions and post-conditions.

Definition 3. The pre-conditions of continueFind ( $p O p$, path, $l$, p):

1) $l$ was child of $p$.

2) $p$ was child of the top node in path if it is not empty.

The post-conditions are the same as the find function.

Definition 4. The pre-conditions of continueFindCommon(il, $r l$, lca, $r O p$, iOp, iPath, rPath, ip, rp, oldKeyX, oldKeyY, newKeyX, newKeyY, iFail, rFail, cFail):

1) At least one of iFail, rFail or cFail is true.

2) il was a child of ip.

3) ip was a child of the top node in iPath if it is not empty.

4) $r l$ was a child of $r p$.

5) rp was a child of the top node in $r$ Path if it is not empty.

The post-conditions are the same as the findCommon function.

We now prove that the continueFind function satisfies its pre-conditions and post-conditions.

Lemma 8. Every call to the continueFind function satisfies its pre-condition and post-conditions.

Proof. The post-conditions:

At line 132 of the continueFind function, it executes the find function. Because the find operation satisfies its post-conditions by Lemma 6 , the continueFind function also satisfies the same conditions.

The pre-condition:

The continueFind function is invoked at line 119 and line 176

Initially, the continueFind function follows the find function at line 106 and line 163 For the first part, the postcondition of the find function shows that $l$ was a child of $p$ which popped at line 109 or line 166.(Lemma 5). For the second part, after popping $p$, it becomes a child of the top node in path if it is not empty.

Otherwise, it reads results from the continueFind invocation at the prior iteration. As the post-conditions of the continueFind function is the same as the find function, we prove the lemma.

We now prove that the continueFindCommon function satisfies its pre-conditions and post-conditions.

Lemma 9. Every call to the continueFindCommon function satisfies its pre-conditions and post-conditions

Proof. The post-conditions:

There are three cases, the first part of the pre-condition shows that it must enter one of the loop. We consider each case by the program execution order. 
1) If $r F a i l$ is true and $c F a i l$ is false, it executes the case starts from line 236. If $c$ Fail is not set to true, it executes the find function at line 302 . Because the find function satisfies its post-conditions (Lemma 6), the lemma holds. If $i F$ ail is true, it comes to the second part of the proof. If $c F a i l$ is set to true, it comes to the third part of the proof.

2) If iFail is true and cFail is false, it executes the case starts from line 235. If $c F$ ail is not set to true, it executes the find function at line 323 . Because the find function satisfies its post-conditions, part 1 and Lemma 6 indicate the Lemma holds. If $c$ Fail is true, it comes to the third part of the proof.

3) If $c F$ ail is true, it executes the case starts from line 240 Because the findCommon function satisfies its post-conditions (Lemma7), it establishes this part of the lemma.

The pre-condition:

The continueFindCommon function is invoked at line 243

For the first condition, line 216, line 217, line 218, line 232 line 235, line 236, line 240 indicate that at least on of three flags is set to true.

For the next conditions, initially the continueFindCommon follows the findCommon function at line 212. As $i p$ and $r p$ are popped at line 213 and line $214, i l$ was a child of $i p$ and $r l$ was a child of $r p$. Further, ip and $r p$ was a child of the top node in $i$ Path and rPath. All these claims are based on Lemma 8 Otherwise, the continueFindCommon function reads results from its invocation at the prior iteration. In such a case, our post-conditions prove the pre-conditions.

We observe that the find function, the continueFind function, the findCommon function, and the continueFindCommon function return a similar pattern of results. The find function and the continueFind function return a tuple $\langle l, p O p, p a t h\rangle$. The findCommon function and the continueFindCommon function return two such tuples. Hence We use find(keys) to denote such a set of find operations for searching keys. Further, we number find(keys) by their invocation orders. Invocations at line 106, line 163 , and line 212 are at iteration $_{0}$. Invocations at line 106, line 163 , and line 212 are at iteration $_{i}, 0<i$.

Definition 5. For compress (path,p) invoked at $T_{i}$, it has following pre-conditions:

1) $p$ is an Internal node.

2) path is read from the result of find(keys) at the prior iteration.

3) If path is not empty, $p$ was a child node of the top node in path at $T_{i 1}<T_{i}$.

Lemma 10. Every call to the compress function satisfies its preconditions.

Proof. The compress function is called at line 171 or line 229 At line 171, $p$ is a node popped from $p a t h$ (line 166 At line 229. $r p$ is an Internal node popped from $r$ Path (line 214 line 305 or line 344 . This proves the first part.
For last two parts, since the compress function reads result following find(keys), it suffices to prove the postconditions of find(keys) satisfy the preconditions. By Lemma 8, Lemma 9. Lemma 6, and Lemma 7, the postconditions of the find function, the findCommon function, the continueFind function, and the continueFindCommon function establish the lemma.

Corollary 2. Every call to the check function satisfies its preconditions that $p$ is an Internal node.

Definition 6. For moved (node) invoked at $T_{i}$, it has following the pre-conditions that node is a Leaf node or an Empty node.

Lemma 11. Every call to the moved function satisfies its precondition.

Proof. The moved function is called at line 108 , line 165 . line 283 , line 280, line 324, line 303

At line 108 it reads $l$ from line 106 or line 119 Hence, according to Lemma $6, l$ is a Leaf node or an Empty node.

At line 165, it reads $l$ from line 163 or line 176 Hence, according to Lemma $6, l$ is a Leaf node or an Empty node.

At line 283 and line 280, it reads $i l$ from line 282 and $r l$ from line 279 Therefore, according to Lemma 6, il and $r l$ are Leaf node or Empty nodes.

At line 324 , it reads $i l$ from line 323 Therefore, according to Lemma 6 il is a Leaf node or an Empty node.

At line 303 , it reads $r l$ from line 302 Therefore, according to Lemma 6rl is a Leaf node or an Empty node.

Based on these post-conditions, we show that each op created at $T_{i}$ store their corresponding information.

Lemma 12. For op created at $T_{i}$ :

1) If op is a Substitute object, op.parent has contained op.oldChild, a Leaf node, at $T_{i 1}<T_{i}$ from the result of find(keys) at the previous iteration.

2) If op is a Compress object, op.grandparent has contained op.parent, an Internal node, at $T_{i 1}<T_{i}$ from the result of find(keys) at the previous iteration.

3) If op is a Move object, op.iParent has contained op.oldIChild, a Leaf node, op.rParent has contained op.oldRChild, a Leaf node, op.iParent has contained op.oldIOp, and op.rParent has contained op.oldROp before $T_{i}$ from the result of find(keys) at the previous iteration.

Proof. 1) A Substitute object is created at line 112 or line 168 by assigning $\langle p, l$, new Node $\rangle$. Since $p$ read from the top node in path at line 109 or line 166 and $l$ is returned by the find function or findCommon function, the post-conditions of them establish the claim according to Lemma 6. Lemma 7. Lemma 9. and Lemma 8

2) A Compress object is created at line 185 by assigning $\langle p a t h, p\rangle . p$ is passed from the top of path at line 166 or line 214 the post-conditions of find(keys) and the property of path establishes the claim (Lemma6.6. Lemma 7, Lemma 9, Lemma 8, Lemma 3, and Lemma 4 .

3) A Move object is created at line 224 by assigning $\langle i p, r p, i l, r l$, new Node, $i$ OldOp, $r O l d O p\rangle$. ip is assigned to the top node of $i$ Path at line 213, $r p$ is 
assigned to the top node of $r P a t h$ at line $214, i l$ was a child of the top node of $i P a t h, r l$ was a child of the top node of $r P a t h$, and $i O p$ and $r O p$ are read from $i p$ and $r p$ by the post-conditions of the findCommon function and the continueFindCommon function (Lemma 9 and Lemma 7

Then we prove the pre-conditions of other functions which use the result of above functions to modify quadtree.

The following lemmas satisfy a basic pre-condition that their arguments are the same type as indicated in algorithms.

Lemma 13. 1) Every call to the help function satisfies its pre-conditions.

2) Every call to the helpSubstitute function satisfies its preconditions.

3) Every call to the helpCompress function satisfies its preconditions.

4) Every call to the helpMove function satisfies its preconditions.

Proof. 1) The help function is called at line 118 line 175, line 311, line 287, line 333, and line 332 At line 118 and line 175, $p O p$ might be obtained from the result of the find function and the continueFind function. Hence, the post-conditions of them ensures that $p O p$ is an Operation object read from $p$. Otherwise, $p O p$ might be obtained from line 116, or line 173 by reading a node's $o p$.

At line 311 and line 287, $r O p$ and $i O p$ are passed the move operation. The post-conditions of the continueFindCommon function and the findCommon function (Lemma 7 and Lemma 9, with line 235 line 236, and line 240 guarantee that arguments' type are the same.

At line 333 and line 332, $r O p$ and $i O p$ could be passed from the move function or read from $i p$ and $r p$ in the continueFindCommon function at lines that have been mentioned.

2) The helpSubstitute function is called at line 114 line 170. and line 150 At line 114 and line 170 op is created at line 112 or line 168 respectively. At line 150, it checks whether op is Substitute before calling the helpSubstitute function.

3) The helpCompress function is called at line 185 line 317, line 297, and line 339. For each invocation, it checks whether $o p$ is Compress beforehand.

4) The helpMove function is invoked at line 228 line 239. and line 151 At line 228 and line 239, op is created at line 224 At line 151, it checks whether $o p$ is Move before the invocation.

Corollary 3. For help $(o p)$, op is read from an Internal node.

Definition 7. For helpFlag $\left(p\right.$, oldOp, newOp) invoked at $T_{i}$, it has the pre-conditions:

1) $p$ is an Internal node.

2) For cflag, $p$ has contained $p O p$ at $T_{i 1}<T_{i}$ after reading $p$; otherwise, $p$ has contained $p O p$ at $T_{i 1}<T_{i}$ from the result of $f$ ind(keys) at the prior iteration.
Lemma 14. Every call to the helpFlag function satisfies its preconditions.

Proof. The helpFlag function is invoked at line 113 line 169 . line 186, line 226, line 248-line 250 and line 261-line 267.

At line 113 and line 169 , the post-conditions of the find function and the continueFind function (Lemma 6 and Lemma 8 imply that $p$ is an Internal node popped at line 109 and has contained $p O p$.

At line 186, the pre-condition of the compress function (Lemma 10 shows that $p$ and nodes from path are read from find (keys) at the prior iteration, and $p O p$ is read at line 181 . Thus, $p$ has $p O p$ at $T_{i 1}<T_{i}$ after reading $p$.

At line 226. $i p$ and $r p$ are popped from $i P a t h$ at line 213 and line 214 or read from the continueFindCommon function. By Lemma 7 and Lemma 9 , either op.iParent has contained op.iOp or op.rParent has contained op.rOp.

At line 248-line 250 and line 261-line 267 by the precondition of the helpMove function 13, op is a Move object. Therefore, according to Lemma 12, either op.iParent has contained op.iOp or op.rParent has contained op.rOp.

Lemma 15. Every call to the hasChild function satisfies its preconditions that parent is an Internal node.

Proof. According to Lemma 11. node is a Leaf node or an Empty node. According to Lemma 14. flag operations only perform on Internal nodes. Therefore line is the only place that set a Move op on a Leaf node. By Observation 1, op is initially null. After checking the condition, $o p$ is assigned to a Leaf node where op.iParent is an Internal node by Lemma 12

Definition 8. For helpReplace (p, oldChild, newChild) invoked at $T_{i}$, it has the pre-conditions:

1) $p$, oldChild, and newChild are read from the same op.

2) newChild is a node that has not been in quadtree.

3) $p$ is an Internal node that has contained oldChild at $T_{i 1}<T_{i}$.

Lemma 16. Every call to the helpReplace function satisfies its pre-conditions.

Proof. For the first condition:

The helpReplace function is invoked at line 145 , line 193 . line 254 259

By Lemma 13, at line 193, it reads a Compress object; at line 145 it reads a Substitute object; at line 254 259 it reads a Move object.

For the second condition:

Since part 1 illustrates that $p$, oldChild, and newChild are read from the same $o p$, if $o p$ is a Substitute object, op.new Node is created at line 111 or line 161; if op is a Compress object, each time the helpCompress function creates a new Empty node at line 193 if $o p$ is a Move object, op.newIChild is created at line 220, and an empty node is created at line 145

For the third condition:

Since part 1 illustrates that $p$, oldChild, and newChild are read from the same $o p$, Lemma 12 shows that $p$ has contained oldChild.

Definition 9. For createNode (l, p, newKeyX, newKeyY, value) that returns a newNode invoked at $T_{i}$, it has the pre-conditions: 
1) $p$ is an Internal node.

2) $p$ has contained $l$ at $T_{i 1}<T_{i}$ from the result of find(keys) at the prior iteration.

post-conditions:

The newNode returned is either a Leaf node with newKey and value, or a sub-tree that contains both l.key node and newKey node with the same parent.

Lemma 17. Every call to the createNode function satisfies its pre-conditions and post-condition.

Proof. The createNode function is invoked at line 111 and line 221

At line 111, the post-conditions of the find function and the continueFind function ensure that $l$ has been a child of $p$ that popped from path at line 109 (Lemma 6 and Lemma 8 .

At line 221 the post-conditions of the findCommon function and the continueFindCommon function ensure that $l$ has been a child of $p$ popped at line 213 (Lemma 7 Lemma 91.

Though we have not presented the details of the createNode function, our implementation guarantee that the post-condition must be satisfied.

\section{.1.2 Flag and replace operations}

We argue that each successful CAS operation occurs in a correct order. At outset, we argue that CAS behaviours for each helpFlag function. A flag operation involves three arguments: node, old $O p$, new $O p$. A replace operation involves three arguments: $p$, oldChild, newChild. From Figure 9. we denote each flag operation accordingly. iflag occurs in the insert operation, rflag occurs in the remove operation, and mflag occurs in the move operation. Moreover, we specify a flag operation which attaches a Clean op on a node as an unflag operation. We call ireplace as the replace operation occurs in the insert operation, rreplace as the replace operation occurs in the remove operation, mreplace as the replace operation occurs in the move operation, and creplace as the replace operation occurs in the compress operation. The next lemmas describe the behaviours of the helpFlag function according to the state transition diagram.

Observation 4. op is only attached on an Internal node.

Lemma 18. For a new node $n$ :

1) It is created with a Clean op.

2) rflag, iflag, mflag or cflag succeeds only if n's op is Clean.

3) unflag succeeds only if n's op is Substitute or Move.

4) Once n's op is Compress, its op will never be changed.

Proof. 1) As shown at line 71. Internal nodes are assigned a Clean op when they are created.

2) Before iflag, op is checked at line 110; before rflag, $o p$ is checked at line 167 before $c$ flag, op is checked at line 182 . For the move operation, before mflag on oldKey's parent and newKey's parent, they are checked at line 217 and line 216

3) unflag is called at line 146 and line 261 line 267 where the pre-conditions specify that $o p$ is Move or Substitute according to Lemma 13.

4) By part 3, there's no unflag operation happens on an Internal node with a Compress op.
Next we illustrate that there's no ABA problem on any $o p$. That is to say, in terms of an Internal node $n$, its $o p_{i}$ at $T_{i}$ has not appeared at $T_{i 1}<T_{i}, o p_{i 1} \neq o p_{i}$. We prove the following lemma:

Lemma 19. For an Internal node $n$, it never reuse an op that has been set previously.

Proof. If a node's $o p$ is set to $o p_{i}$ at $T_{i}$, it has never been appeared before. We prove the lemma by discussing different types of flag operations.

For iflag, a Substitute op is created at line 112 before its invocation. For rflag, a Substitute op is created at line 168 before its invocation. For a $c f l a g$, a Compress $o p$ is created at line 185 before its invocation. For mflag, a Move op is created at line 224 before its invocation. Because each new $O p$ is newly created, it has not been set before. For unflag, each time it creates a new Clean op to replace the prior Move op or Substitute op by Lemma 18. Therefore, the new Clean op has never appeared before.

We have shown that for each successful flag operation, it never set an $o p$ that has been used before. Every Internal node is initialized with Clean op by Lemma 18 and we use a flag operation to change $o p$ at the beginning of each CAS transition. We say successive replace operations and unflag operations that read the $o p$ belongs to it. According to Figure 9 there are threes categories of successful CAS transitions. We denote them as flag $\rightarrow$ unflag, flag $\rightarrow$ replace, and flag $\rightarrow$ replace $\rightarrow$ unflag.

Let flag $_{0}, f l a g_{1}, \ldots$, flag $_{n}$ be a sequence of successful flag operations; let unflag unflag $_{1}, \ldots$, unflag $_{n}$ be a sequence of successful unflag operations. flag $_{i}$ attaches $o p_{i}$, and replace ${ }_{i}$ and unflag $_{i}$ read $o p_{i}$ and come after it. Therefore we say flag $_{i}$, replace $_{i}$, and unflag $_{i}$ belong to the same $o p$. In addition, if there are more than one replace operations belong to the same $o p_{i}$, we denote them as replace ${ }_{i}^{0}$, replace $_{i}^{1}, \ldots$, replace $_{i}^{k}$ ordered by their successful sequence. Similarly, if there are more than one flag operations that belong to $o p_{i}$ on different nodes, we denote them as $f l a g_{i}^{0}$, $\operatorname{flag}_{i}^{1}, \ldots$, flag $_{i}^{k}$. A similar notation is used for unflag . $^{2}$ The following lemmas prove the correct ordering of three different transitions.

Lemma $20 . \quad 1$ 1) ireplace will not be done before the success of iflag which belongs to the same op.

2) rreplace will not be done before the success of rflag which belongs to the same op.

Proof. For the insert operation and the remove operation, at line 145 the helpReplace function is wrapped by the helpSubstitute function. The helpSubstitute function is called at line 114, line 170 and line 150. At line 114 it is called after the success of iflag at line 113. At line 170, it is called after the success of rflag at line 169 . At line 150. by Corollary 3 and Lemma 18, there must have been iflag or rflag that change the node's op from Clean to Substitute.

Lemma 21. creplace will not be done before the success of cflag which belongs to the same op.

Proof. The helpCompress function which wraps creplace is called at line 185, line 317, line 297, and line 339, and 
line 149 At line 185 the helpCompress function follows the successful helpFlag function at line 186 . At line 317 line 297. and line 339, a $p O p$ is read from Internal nodes. At line 149. Lemma 3 shows that $o p$ is read from an Internal node. Hence, by Lemma 18 , successful cflag must have changed the node's op from Clean to Compress.

Lemma 22. mreplace will not be done before the success of $m$ flag which belongs to the same op.

Proof. The helpMove function which wraps two mreplaces is called at line 228, line 239, and line 151. At line 228 and line 239. the Move op is created at line 267 Otherwise, at line 151. by Corollary 3, op is read from an Internal node. Hence, Lemma 18 demonstrates that a successful mflag must have changed the node's op from Clean to Move.

From the above lemmas, we have the following corollary:

Corollary 4. A successful replace operation which belongs to an op will not succeed before it's flag operations have been done.

We discuss each CAS transition accordingly. We begin by discussing the flag $\rightarrow$ replace $\rightarrow$ unflag transition. We refer $\langle$ oldIOp, oldIChild, newIChild $\rangle$ and $\langle$ oldROp, oldRChild, newRChild $\rangle$ to different tuples of $\langle$ oldOp, oldChild, newChild $\rangle$.

Lemma 23. flag $\rightarrow$ replace $\rightarrow$ unflag occurs when $r$ flag $_{i}$,

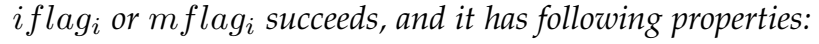

1) replace $_{i}$ never occurs before flag . $_{\text {. }}$

2) flag $_{i}^{k}, 0 \leq k<\mid$ flag $_{i} \mid$ is the first successful flag operation on op $p_{i}$.parent $t^{k}$ after $T_{i 1}$ when $p O p_{i}$ is read.

3) replace $_{i}^{k}, 0 \leq k<\mid$ replace $_{i} \mid$ is the first successful replace operation on op $p_{i}$.parent ${ }^{k}$ after $T_{i 2}$ when op . oldChild $^{k}$ is read.

4) replace $_{i}^{k}, 0 \leq k<\mid$ replace $_{i} \mid$ is the first successful replace operation on op ${ }_{i}$.parent ${ }^{k}$ that belongs to op . $_{i}$.

5) unflag ${ }_{i}^{k}, 0 \leq k<\mid$ unflag $_{i} \mid$ is the first successful unflag operation on op . parent $^{k}$ after flag ${ }_{i}^{k}$.

6) There is no successful unflag operation occurs before replace . $_{\text {. }}$

7) The first replace operation on op p parent $^{k}$ that belongs to op $i$ must succeed.

Proof. 1) Corollary 4 proves the claims.

2) By Lemma 14, for each flag operation, $p$ has contained $p O p$ at some time $T_{i 1}$. Suppose that there's another flag $^{k}$ that occurs after $T_{i 1}$ but before $f l a g_{i}^{k}$, then $f l a g_{i}^{k}$ fails because Lemma 19 shows that $o p$ is not reused. Therefore, it contradicts to the definition that $f l a g_{i}^{k}$ is a successful flag operation.

3) By Lemma 16, for each replace operation, $p$ has contained oldChild at some time $T_{i 1}$. Suppose that there's another replace that occurs after $T_{i 1}$ but before replace ${ }_{i}^{k}$, then replace ${ }_{i}^{k}$ fails because Lemma 16 shows that newChild is never a node in the tree. Therefore, it contradicts to the definition that replace $e_{i}^{k}$ is a successful flag operation.

4) Suppose there's replace ${ }^{k}$ that belongs to $o p_{i}$ and occurs before creplace $e_{\text {. }}^{k}$ It must follow flag according to Corollary 4 If flag $^{k}$ happens before $f l a g_{i}^{k}$, it fails because $f_{a i l}^{k}$ will fail by consequence
(Lemma 19). If $\mathrm{flag}^{k}$ happens after $f l a g_{i}^{k}$, it contradicts Lemma 18 that a flag operation starts Because flag $^{k}$ is done after reading $o p_{i}$.oldChild ${ }^{k}$, replace $^{k}$ is also after reading it. Therefore, if flag $^{k}$ succeeds, it contradicts part 3 that replace ${ }_{i}^{k}$ is the first successful replace operation after reading $o p_{i}$.oldChild ${ }^{k}$.

5) Based on Lemma 14, if there is another unflag changes $p . o p$, unflag $g_{i}^{k}$ that use $o p_{i}$ as the oldOp will fail. This contradicts to the definition that unflag $_{i}^{k}$ is a successful unflag operation that reads $o p_{i}$.

6) We consider each operation separately.

For the insert operation and the remove operation, unflag follows replace immediately at line 146 Assuming that replace ${ }_{i}^{k}$ occurs after unflag $g_{i}^{k}$, there must be another replace reads $o p_{i}$ and changes the link. It contradicts part 3 of the proof that replace ${ }_{i}^{k}$ is the first successful replace operation that belongs to $o p_{i}$.

For the move operation, because we assume that replace operations exist, $d o C A S$ is set to true before performing replace operations. An unflag operation follows replace operations from line 261 267 Consider if a replace ${ }_{i}^{k}, 0 \leq k<\mid$ replace $_{i} \mid$ happens after any unflag operation, then another replace which use the same $o p$ must have succeed as the program order specifies that replace execute before unflag for a process. Hence, replace ${ }_{i}^{k}$ fails and contradicts the definition.

Therefore all successful unflag operations occur after replace.

7) According to Corollary 4 replace ${ }_{i}^{k}$ follows flag ${ }_{i}^{k}$ belongs to $o p_{i}$. Suppose there exists replace after reading $o p_{i}$.parent ${ }^{k}$ and before replace ${ }_{i}^{k}$. Then, there must be flag that precedes replace by Corollary 4 By part 1, flag $_{i}$ is the first successful flag operation after reading $o p_{i}$. By Lemma 14. $o p_{i}$ is read before $o p_{i} . l$. Hence, there does not exist any replace operation between $T_{i 1}$ reading op .oldChild $^{k}$ and flag $_{i}^{k}$. If flag happens between flag $_{i}^{k}$ and replace $_{i}^{k}$, it will also fail because $o p_{i}$.parent ${ }^{k} . o p$ is not Clean. Then, the first replace operation that belongs to $o p_{i}$ must succeed.

We then discuss the ordering of the flag $\rightarrow$ replace transition.

Lemma 24. flag $\rightarrow$ replace occurs only when cflag $_{i}$ succeeds, it has following properties:

1) creplace $_{i}$ never occurs before cflag $_{i}$.

2) cflag $_{i}$ is the first successful flag operation on op $p_{i}$.parent after $T_{i 1}$ when $r O p_{i}$ is read.

3) creplace $_{i}$ is the first successful replace operation on $o p_{i}$.grandparent after $T_{i 1}$ when op p $_{i}$.parent is read.

4) creplace $_{i}$ is the first successful replace operation on $o p_{i}$.grandparent that belongs to op . $_{\text {. }}$

5) There is no unflag operation after creplace . $_{\text {. }}$

6) The first replace operation that belongs to $o p_{i}$ must succeed.

Proof. 1) Corollary 4 proves the claims. 
2) By Lemma 14 for each flag operation, $p$ has contained $p O p$ at some time $T_{i 1}$. Suppose that there's another $c$ flag that occurs after $T_{i 1}$ but before cflag $_{i}$, then $c$ flag $g_{i}$ fails because Lemma 19 shows that op is not reused. Therefore, it contradicts to the definition that $\mathrm{cflag}_{i}$ is a successful flag operation.

3) By Lemma 16. for each replace operation, $p$ has contained oldChild at some time $T_{i 1}$. Suppose that there's another creplace that occurs after $T_{i 1}$ but before replace $_{i}$, then creplace $_{i}$ fails because oldChild remains the same but the link has been changed to another node. Therefore, it contradicts to the definition that creplace $_{i}$ is a successful flag operation.

4) Suppose there's a replace operation that belongs to $o p_{i}$ and occurs before creplace $_{i}$. It must follow cflag according to Corollary 4 If cflag happens before $c f l a g_{i}$, it fails because $c f a i l_{i}$ will fail by consequence (Lemma 19 If cflag happens after cflag $_{i}$, it contradicts Lemma 18 that a flag operation starts with a Clean op. Therefore, cflag is the same as $c$ flag . Because $c$ flag is done after reading $o p_{i}$.parent, creplace is also after reading it. Therefore, if cflag succeeds, it contradicts part 3 that creplace $_{i}$ is the first successful replace operation after reading $o p_{i}$.parent.

5) By Lemma 18, once a node's op is Compress, it will never be changed.

6) Suppose the first replace operation that belongs to $o p_{i}$ fails, there must exist some successful replace after reading $o p_{i}$.parent and before creplace $_{i}$. Further, there is flag that precedes replace by Corollary 4 . If flag happens between creplace $_{i}$ and cflag $_{i}$, flag will fail by Lemma 18 If flag happens between reading $o p_{i}$ and $c$ flagi, it contradicts part 1 that cflag $_{i}$ is the first successful flag operation after reading $o p_{i}$. Because $o p_{i}$ is read after $o p_{i}$.parent, we also have to consider if flag happens before reading $o p_{i}$. Consider if cflag happens, flag $_{i}$ will fail because $o p_{i}$.parent.op will not be set back to Clean according to the fourth part. Consider if mflag, iflag or rflag happens, it will not change the link from $o p_{i}$. grandparent to $o p_{i} . p$ according to Lemma 23

Corollary 5. The first replace operation belongs to op $p_{i}$ must succeed.

Next we discuss the flag $\rightarrow$ unflag transition, where replace operation occurs between a successful flag operation and an unflag operation that belong to the same op. We suppose that op.iFirst is true. The claim for op.iFirst = false is symmetric.

Observation 5. allFlag and iFirst in a Move op are initially false, and they will never be set false after assigning to true.

Lemma 25. For flag $\rightarrow$ unflag circle, it only results from a Move object $o p_{i}$ such that:

1) unflag is the first successful unflag operation on op . $_{\text {. Parent. }}$.

2) The first flag operation on $o p_{i} . i$ Parent must fail, and no later flag operation will succeed.
3) $o p_{i} . i$ Parent and $o p_{i} . r$ Parent are different.

Proof. 1) We prove it by contradiction. Suppose there's another unflag operation that reads $o p_{i}$ and succeeds before unflag $_{i}$ on $o p_{i}$.rParent. It must have changed $o p_{i} . r$ Parent.op to a new $o p$, by Lemma 19 unflag $_{i}$ will fail. This contradicts the definition of unflagi.

2) For the former part of the claim, we prove it by contradiction. Assuming that $o p_{i} . i$ Parent is flagged on $o p_{i}$, and will be unflagged without performing replace operations. However, unflag operations occur after the judgement at line 254 The first process must set $d o C A S$ to true. In the next step, it executes line 254 259 According to Corollary 5, the first replace $_{i}^{t}$ must succeed. Therefore it contradicts the definition that there's no replace operation before an unflag operation. For the latter part, by Lemma 12 $o p_{i}$. iParent has contained $o p_{i} . o l d I O p$. Because the first flag operation on $o p_{i} . i$ Parent fails, latter flag operations read the $o p_{i}$.oldIOp also fail.

3) Assuming $o p_{i} . i$ Parent and $o p_{i} . r$ Parent are the same. By part 2, flag operations on $o p_{i}$. iParent must fail, hence $\operatorname{doCAS}$ will never be true. $o p_{i}$.allFlag is always false by by Observation 5 In this way, line 261267 prevents any unflag operation. This derives a contradiction.

\section{.1.3 Quadtree properties}

In the Section, we use above lemmas to show that quadtree's properties are maintained during concurrent modifications.

Definition 10. Our quadtree has these properties:

1) Two layers of dummy Internal nodes are never changed.

2) An Internal node $n$ has four children, which locate in the direction $d \in\{n w, n e, s w$, se $\}$ respectively according to their $\langle x, y, w, h\rangle$, or $\langle k e y X, k e y Y\rangle$.

For Internal nodes reside on four directions:

- $\quad n . n w \cdot x=n . x, n . n w . y=n . y$;

- $\quad$ n.ne. $x=n . x+w / 2, n . n e . y=n . y$;

- $\quad n . s w . x=n . x, n . s w . y=n . y+n . h / 2$;

- $\quad$ n.se. $x=n . x+w / 2, n . s e . y=n . y+h / 2$,

and all children have their $w^{\prime}=n . w / 2, h^{\prime}=n . h / 2$.

For Leaf nodes reside on four directions:

- $n . x \leq n . n w . k e y X<n . x+n . w / 2, n . y \leq$ n.nw.key $Y<n . y+n . h / 2$;

- n.x+n.w/2 $\leq$ n.ne.keyX $<$ n.x + n.w, n.y $\leq$ n.ne.key $Y<n . y+n . h / 2$;

- $n . x \leq n . s w . k e y X<n . x+n . w / 2, n . y+$ $n . h / 2 \leq n . s w . k e y Y<n . y+n . h$;

- $\quad n . x+n . w / 2 \leq n . s e . k e y X<n . x+n . w, n . y+$ $n . h / 2 \leq n . s e . k e y Y<n . y+n . h$.

Lemma 26. Two layers of dummy nodes are never changed.

Proof. We prove the lemma by induction. Only replace operations will affect the structure of quadtree as we define in Definition 10 .

Initially the property holds as we initialize quadtree with two layers of dummy nodes and a layer of Empty nodes. 
Suppose that after replace $_{i}$ the lemma holds, we shall prove that after replace $_{i+1}$ the lemma is still true. For ireplace, rrepalce, and mreplace, the pre-condition 16 ensures that three nodes are from the same op. Thus, as Lemma 12 further ensures that they only swing Leaf nodes and Empty node, dummy node are not changed. For creplace, since at replace $i$ the property is true, the root is connected to a layer of dummy nodes. Lemma 12 shows that op.oldChild is an Internal node, and line 186 shows that the root node's child pointer will never be changed. Therefore, replace $_{i+1}$ will not occur on the root node.

Lemma 27. Children of a node with Compress op will not be changed.

Proof. Based on Lemma 18, the node's op will never be changed after being set to Compress. Lemma 24 and Lemma 23 indicate that for a node flagged with Compress op, only itself will be unlinked. This establishes the Lemma.

Lemma 28. Only an Internal node with all children Empty could be attached with a Compress object.

Proof. Corollary 2 ensures that the pre-conditions of the check function is satisfied. It checks whether all children are Empty before calling the helpFlag function at line 186 This establishes the Lemma.

Lemma 29. An Internal node whose op is not Compress is reachable from the root.

Proof. We have to consider all kinds of replace operations.

For ireplace, rrepalce, and mreplace, by Lemma 16 and Lemma 12 they use a new node to replace a Leaf node or an Empty node. Thus, Internal nodes are still reachable from the root.

For creplace, by Lemma 16 and Lemma 12, it replaces Internal nodes. Lemma 24 shows that it is preceded by cflag. Lemma 28 implies that all children are Empty nodes. Therefore, an Internal node with a Compress op and its children are not reachable from the root only after the success of creplace.

Next we define active set and inactive set for different kinds of nodes. We say a node is moved if the moved function turns true. For an Internal node or an Empty node, if it is reachable from the root in snapshot $T_{i}$, it is active; otherwise, it is inactive. For a Leaf node, if it is reachable from the root in snapshot $T_{i}$ and not moved, it is active; otherwise, it is inactive. We denote path $\left(\right.$ keys $\left.^{k}\right), 0 \leq k<\mid$ replace $_{i} \mid$ as a stack of nodes pushed by find(keys) in a snapshot. We define physical_path $\left(k_{e y s}{ }^{k}\right)$ to be the path for keys ${ }^{k}$ in snapshot $T_{i}$, consisting of a sequence of Internal nodes with a Leaf node or an Empty node at the end, which is the actual path in the snapshot. We say a subpath of path $\left(\right.$ keys $\left.^{k}\right)$ is an active_path if all nodes from the root to node $n \in \operatorname{path}\left(\right.$ keys $\left.^{k}\right)$ are active due to their pushing order. Hence a physical path with a Leaf node is active only if $\operatorname{path}\left(k_{e y s^{k}}\right)$ is an active path and the Leaf node is active.

Lemma 30. There's at most a node with Compress op in the subpath of path $\left(k^{2 e y s}{ }^{k}\right)$ that is active, and it resides in the end of the path if exists.
Proof. Assume that there are two nodes $n$ and $n^{\prime}$ reside in the active path $\left(k_{e y s}{ }^{k}\right)$ with Compress op. We prove the lemma by contradiction. Because $n$ and $n^{\prime}$ are active with Compress op, all children of $n$ and $n^{\prime}$ are Empty (Lemma 28 Since $n$ and $n^{\prime}$ are Internal nodes in the same path, it derives a contradiction.

For the second part of the proof, if $n$ resides in other places in path, its children should be Empty by Lemma 28. Therefore, only if $n$ is on the end of path, conditions are satisfied.

Lemma 31. For path $\left(\right.$ keys $\left.^{k}\right)$, if $n_{t}$ is active in snapshot ${ }_{T_{i}}$, then $n_{0}, \ldots, n_{t-1}$ above $n_{t}$ are active.

Proof. By Lemma 29 , nodes with op other than Compress are reachable from the root. Lemma 30 indicates that a node with a Compress op is on the end of the path. Therefore, other nodes do not have a Compress op, which establishes the lemma.

Lemma 32. If in the snapshot $T_{i}, n$ is the LCA on active_path for oldKey and newKey. Then at $T_{i 1}, T_{i 1}>T_{i}, n$ is still the LCA on active_path for both oldKey and newKey if it is active.

Proof. Nodes from root to the LCA node shares a common path (Observation 1 Because the LCA node is active, nodes above the LCA node are active. Hence, the subpath from the root to the LCA node is active by Lemma 31

Lemma 33. Nodes a with a Compress op will not be pushed into path more than once.

Proof. Supposing that a node $n$ is active in snapshot $_{T_{i}}$, and it becomes inactive at $T_{i 1}>T_{i}$. Besides, suppose that op of $n$ is Compress and pushed at $T_{i 2}, T_{i}<T_{i 2}<T_{i 1}$. We prove that after $T_{i 2}, n$ will never be pushed again.

Lemma 24 shows that a node with a Compress op will never be unflagged. Hence, based on Lemma 6 and Lemma 6, we have to prove that nodes in path with a Compress op will not be pushed into again. For the insert operation and the remove operation, $p O p$ is checked at line 123 and line 127 before the next find operation. For the move operation, $r \mathrm{Op}$ is checked at line 312, line 316. line 339. and $i O p$ is checked at line 296 and line 288 before calling the findCommon function and the find function. Hence, at $T_{i 2}<T_{i 1}$, op must be checked before find(keys) so that nodes with a Compress op will not be pushed more than once.

Lemma 30 shows that for active_path, active nodes with a Compress op will only reside at the end. If other nodes pushed before $n$ will become inactive, we can pop them beforehand until reaching the last node an op other than Compress.

Lemma 34. After the invocation of find(keys) which reads $l^{t}$, there is a snapshot, where the path from the root to $l^{t}$ is physical_path $\left(\right.$ keys $\left.^{t}\right)$.

Proof. We prove the lemma by induction.

In the base case, where path starts from the root node, the claim is true.

We consider the pushing sequence as $n_{0}, n_{1}, \ldots, n_{k}$. Suppose that for first $k$ nodes, the path from the root to $n_{k}$ is 
the physical_path. We shall prove that for $n_{k+1}$, the lemma is true. If there is no replace operation before reading $n_{k+1}$, it is obvious that we can linearized it as the same snapshot before pushing $n_{k}$.

Next, we assume that replace occurs on $n_{k}$ before reading $n_{k+1}$, and results in snapshot. There are two cases:

1) Consider if replace occurs after reading $n_{k+1}$. We could have snapshot $_{k+1}=$ snapshot $_{k}$ because $n_{k+1}$ is connected to an active node in a snapshot so that $n_{k+1}$ is also reachable from the root.

2) Consider if replace occurs before reading $n_{k+1}$. If the replace operation changes $n_{k}$, then $n_{k}$ is flagged with a Compress op by Lemma 24 Then we have snapshot $_{k+1}=$ snapshot $_{k}$ because Lemma $27_{27}$ demonstrates that a node flagged with a Compress has no successive replace operations on its child pointers. Otherwise, if the replace operation changes $n_{k+1}, n_{k}$ is not changed by Lemma 23 Hence, we have snapshot $_{k+1}=$ snapshot, where $n_{k+1}$ is reachable from the root in the snapshot just after a replace operation.

Lemma 35. After ireplace, rreplace, mreplace, and creplace, quadtree's properties remain.

Proof. We shall prove that in any snapshot $_{T_{i}}$, quadtree's properties remain.

First, Lemma 10 shows that two layers of dummy nodes remain in the tree. We have to consider other layers of nodes which are changed by replace operations.

Consider creplace that replaces an Internal node by an Empty node. Because the Internal node has been flagged on a Compress op before creplace (Lemma 24), all of its children are Empty and not changed (Lemma 28 and Lemma 27). Thus, creplace does not affect the second claim of Definition 10

Consider ireplace, rreplace, or mreplace that replaces a terminal node by an Empty node, a Leaf node, or a sub-tree. By Lemma 23, before replace ${ }^{k}$, op.parent ${ }^{k}$ is flagged with op such that no successful replace operation could happen on op.parent ${ }^{k}$. Therefore, if the new node is Empty, it does not affect the tree property. If the new node is a Leaf node or a sub-tree, based on the post-conditions of the createNode function (Lemma 17), after replace operations the second claim of Definition 10 still holds.

\section{.2 Linearizability}

In this Section, we define linearization points for basic operations. As the compress operation is included in the move operation and the remove operation that returns true, it does not affect the linearization points of them. If an algorithm is linearizable, it could be ordered equivalently as a sequential one by their linearization points. Since all the modifications depend on find(keys), we first point out its linearization point.

To prove that our linearization points are correct, we shall demonstrate that for some time $T_{i}$, the key set in snapshot $_{T_{i}}$ is the same as the results of modifications linearized before $T_{i}$. For find(keys), we should define the linearization point at some snapshot $_{T_{i}}$ so that $l^{t}$ returned is on the pysical_path for $k e y^{k}$ in snapshot $_{T_{i}}$.

Lemma 36. For find(keys) that returns tuples $\left\langle l^{k}, p O p^{k}, p^{2} h^{k}\right\rangle$, there's a snapshot after its invocation and before reading $l^{k}$, such that path $\left(\right.$ keys $\left.{ }^{k}\right)$ returned with $l^{k}$ at the end is a physical_path in snapshot $T_{T_{i}}$.

Proof. Since $l^{k}$ is a Leaf node or an Empty node, by Lemma 34 there is a snapshot $T_{i}$ that $l^{k}$ as the last node from $l_{i-1}$, and $l_{0}$ to $n_{i-1}$ in the path $\left(\right.$ keys $\left.^{k}\right)$ are active. Therefore path $\left(k e y s^{k}\right)$ with $l^{k}$ is physical_path $\left(\right.$ keys $\left.^{k}\right)$ in snapshot $_{T_{i}}$. We define snapshot $_{T_{i}}$ as $\operatorname{find}\left(k_{\text {eys }}{ }^{k}\right)^{\prime}$ 's linearization point.

In the next, we define linearization points for basic operations. For the insert operation, the remove operation, and the move operation that returns true, we define it to be the first replace $_{i}^{k}, 0 \leq k<\mid$ replace $_{i} \mid$ belongs to $o p_{i}$. To make a reasonable demonstration, we first show that the first replace $_{i}^{k}$ belongs the $o p_{i}$ created by each operation itself, and then illustrate that $o p$ is unique for each operation.

Lemma 37. If the insert operation, the remove operation, or the move operation that returns true, the first replace ${ }_{i}^{k}$ occurs before the returning, and it belongs to op $p_{i}$ created by the operation itself.

Proof. First we prove that replace $e_{i}^{k}$ belongs to $o p_{i}$ created by the operation itself.

For the insert operation, it returns true after successful iflag and ireplace using op created at line 112

For the remove operation, it returns true after successful rflag and rreplace using op created at line 168

For the move operation, it returns true after successful mflag and mreplace using op created at line 224.

Corollary 5 indicates that the first replace operation must succeed, therefore all successful replace operations happen before returning.

Then, we define the linearization points of the insert operation, the remove operation, and the move operation that returns true are before returning. We should also point out that there's only one op lead to the linearization point for each operation.

Lemma 38. For the insert operation, the remove operation, and the move operation that returns true, op is created at the last iteration in the while loop.

Proof. Based on Lemma 23, all successful insert, remove and move operations follow the flag $\rightarrow$ replace $\rightarrow$ unflag transition. flag is the first successful flag operation after reading $o p_{i}$.parent, so there is no other flag operation further. This establishes the claim.

Lemma 39. If the insert operation, the remove operation, and the move operation that return false, successful replace happens before returning.

Proof. As Lemma 38 points out that op is created at the last iteration. Moreover, before returning true, $\mathrm{mflag}$, iflag and $r$ flag must succeed according to the program order. As the first replace operation that belongs to $o p_{i}$ must succeed (Lemma 5, replace happens before returning. 
Lemma 40. If the insert operation, the remove operation, and the move operation that return false, there's no successful replace ever happened.

Proof. Consider the execution of the insert operation, it returns false at line 108 where op created are not flagged on nodes. Consider the remove operation, it returns false at line 165. Also, op created are not flagged on nodes. For the move operation, it returns false by calling the findCommon function or the continueFindCommon function so that the helpMove function must fail or it is not called. If the helpMove function is not called, there is no replace operations. If the helpMove function returns false, by the ordering of mflag and unflag (Lemma 23 and Lemma 25), op.iParent and op.rParent are different. Moreover, the flag operation on the latter parent will fail such that $d o C A S$ is false and no replace operation will perform.

In the next step, we show that how the algorithm could be ordered as a sequential execution. First we linearize the insert operation, the remove operation and the move operation that returns true, where replace results in changing the key set. Let $s_{i}$ be a set of Leaf nodes that are active in snapshot $_{T_{i}}$ after performing operations $o_{0}, o_{1}, \ldots, o_{n}$, ordered by their linearization points replace $_{0}$, repalce $_{1}, \ldots$, replace $_{n}$ sequentially.

Observation 6. If a Leaf node is moved, its op is set before the replace operation on op.iParent, which is before the replace operation on op.rParent.

Lemma 41. For the contain operation that returns true, there is a corresponding snapshot that $l^{k}$ is active in snapshot ${ }_{T_{i}}$. For the contain operation that returns false, there is a corresponding snapshot that $l^{k}$ is inactive in snapshot $T_{i}$.

Proof. For the first part, we prove that in a snapshot, the end node $l^{k}$ contains $k e y s^{k}$ and is not moved. Lemma 36 shows that there is a snapshot consists of physical_path $\left(\right.$ keys $\left.^{k}\right)$ after calling, thus $l^{k}$ that contains keys ${ }^{k}$ is in snapshot $_{T_{i}}$ before judging whether the node is moved. We only have to discuss mreplace in the next cases because other replace operations will not affect $l^{k} . o p$. If $l^{k} . o p$ is null at verifying, mreplace must have not been done according to Observation 6 In this case, we could linearize it at snapshot $_{T_{i}}$. If $l^{k} . o p$ is not null, but mreplace occurs after verifying whether l.op.iParent has l.op.oldIChild, then it is also before the second mreplace on l.op.r Parent (Observation 6). We could also linearize it snapshot $_{T_{i}}$.

For the second part, Lemma 36 shows that there is a snapshot consists physical_path $\left(\right.$ keys $\left.^{k}\right)$. By Lemma 35, in every snapshot our quadtree's property maintains. Hence, if $l^{k}$ does not contain $k e y s^{k}$, we could linearize it at snapshot $_{T_{i}}$. Or else, if $l^{k}$ is in physical_path, but $l^{k}$ is moved at verifying, we could linearize at snapshot $_{T_{i 1}}$ after mreplace.

Lemma 42. 1) For the insert operation, it returns true when key $\notin s_{i-1}, k e y \in s_{i}$.

2) For the remove operation, it returns true and key $\in$ $s_{i-1}, k e y \notin s_{i}$.

3) For the move operation, it returns true and oldKey $\in$ $s_{i-1}$, newKey $\notin s_{i-1}$, oldKey $\notin s_{i}$, and newKey $\in$ $s_{i}$.
Proof. Lemma 39 shows that there is successful replace occurs before the insert operation, the remove operation, and the move operation that returns true.

If a replace operation succeeds, op.parent has been flagged with op other than Compress before replace (Lemma 24 and Lemma 23. Lemma 29 implies that op.parent is reachable from the root. Therefore, it is active. Besides, Corollary 31 shows that the path from the root to op.parent is an active. Therefore a snapshot exists such that the path from the root to op.oldChild is a physical_path(key).

First we prove that $k e y \notin s_{i-1}$. By Lemma 41 , in the snapshot $l$ is inactive. Otherwise, replace operations will not execute. Hence, $k e y \notin s_{i-1}$.

Second, by the post-conditions of the createNode function 17, it creates a node or a sub-tree that contains newKey. According to Corollary 35, after ireplace the node contains newKey is inserted and quadtree's properties maintained. Hence, key $\in s_{i}$.

2) First we prove that key $\in s_{i}$. By Lemma 41, in the snapshot $l$ is active. Otherwise, replace operations will not execute. Hence, key $\notin s_{i-1}$.

Second, the remove operation creates an Empty node. According to Corollary 35, after rreplace the node contains oldKey is removed so that key $\in s_{i}$.

3) First we prove that oldKey $\in s_{i-1}$, newKey $\notin s_{i-1}$. By Lemma 41, $r l$ is active at snapshot $_{T_{i}}$, $i l$ is inactive at snapshot $_{T_{i 1}}, T_{i}<T_{i 1}$. We have to demonstrate that $r l$ is still active at snapshot $_{T_{i 1}}$ so that we can use snapshot $_{T_{i 1}}$ as $s_{i-1}$.

We prove it by contradiction. Assuming that at snapshot $_{T_{i 1}} r l$ is inactive, hence there's some replace operation happens before $T_{i 1}$ and after $T_{i}$. However, based on Lemma 23, replace $_{i}$ is the first successful replace operation that after reading $r l$. Therefore, it derives a contradiction. Hence we prove that in snapshot $_{T_{i 1}} r l$ is still active.

Finally we order linearization points for the insert operation, the remove operation and the move operation that returns false. We also order the contain operation. We consider they are linearized between replace $_{i-1}$ and replace $_{i}$ if exists.

Lemma 43. 1) For the insert operation returns false, $k e y \in s_{i-1}$.

2) For the remove operation returns false, key $\notin s_{i-1}$.

3) For the move operation returns false, either oldKey $\notin$ $s_{i-1}$ or new Key $\in s_{i-1}$.

Proof. The first two parts are equivalent as the case in Lemma 41

Lemma 44. Our quadboost is a linearizable implementation.

Proof. By lemma 42 and Lemma 43 , our algorithm returns the same result as they are finished in the linearized order. Therefore we prove our algorithm is linearizable and our linearization points are correct. 


\section{.3 Progress Condition}

We say an algorithm is non-blocking if the system as a whole is making progress even if some threads are starving. We prove our quadboost is non-blocking by following set of lemmas. We assume that there are finite number of basic operations invocations.

Observation 7. There are finite number of basic operations.

Lemma 45. path $\left(k e y^{k}\right)$ returned by find(keys) consists of finite number of keys.

Proof. Observation 7 shows that the number of successful insert operation and move operation is limited. Lemma 40 illustrates that for basic operations return false, there is no successful replace operation. Hence, only successful insert and move operations add nodes into quadtree. The postconditions of the createNode function (Lemma 17) and the effects of replace (Lemma 35) demonstrate that they will add finite number of nodes into quadtree. We then prove that $p a t h^{k}$ is terminable. By Lemma 34 find(keys) returns $\operatorname{path}\left(k e y^{k}\right)$ which is a subpath of physical_path $\left(k e y s^{k}\right)$ in snapshot $_{T_{i}}$ which is terminable. Thus, there are finite number of nodes in path ${ }^{k}$.

Corollary 6. The compress function must terminate.

Proof. By Lemma 45 path ${ }^{k}$ is terminable. Since other functions called by the compress function do not consist loops, the compress function must terminate.

The we shall demonstrate that there are finite number of three different CAS transitions.

Lemma 46. There is an unique spatial order among nodes in quadtree in snapshpt $T_{i}$

Proof. As illustrated by the algorithm at line 223 , the getSpatialOrder function compares $i p$ with $r p$ by the order: $x \rightarrow$ $y \rightarrow w$. In our quadtree, we only consider square partitions on two-dimensional space. Hence, $w$ is always equal to $h$. We prove the lemma by contradiction. Assuming there are two different Internal nodes with the same $\langle x, y, w\rangle$, they represent the same square starting with left coroner $\langle x, y\rangle$ with width $w$. By Lemma 35 , in every snapshot quadtree's properties remain. There cannot be two squares with the same left corner and $w$. Hence, in snapshpt $t_{T_{i}}$, a Internal node consists of a unique $\langle x, y, w\rangle$ tuple that can be ordered correctly.

Lemma 47. 1) There are finite number of successful flag $\rightarrow$ replace $\rightarrow$ unflag transitions.

2) There are finite number of successful flag $\rightarrow$ replace transitions.

3) There are finite number of successful flag $\rightarrow$ unflag transitions.

Proof. 1) By Lemma 39 , replace operations only occur in basic operations that return true. By Lemma 38 . there's a unique op created for operations that return true. Hence, for the move operation, the insert operation and the remove operation, there are finite number of $f$ lag $\rightarrow$ replace $\rightarrow$ circles.

2) By Lemma 39. replace operations only occur in basic operations that return true. Lemma 24 shows that the flag $\rightarrow$ replace transition happens only in the compress function which is called by the successful remove operation or the move operation. Hence, there are a finite number of flag $\rightarrow$ replace transitions, as Corollary 6 shows that the compress function is terminable.

3) The flag $\rightarrow$ unflag transition executes only in the helpMove function where op.iParent cannot be flagged according to Lemma 25 (if op.iFirst is true). The case that op.iFirst is false can be proved symmetrically.

From first two parts of the lemma, there are finite number of replace operations that change quadtree's structure. If the flag $\rightarrow$ replace $\rightarrow$ transition happens simultaneously, it will reset a node's op to Clean. If the flag $\rightarrow$ replace $\rightarrow$ unflag transition happens in the meantime. Lemma 33 indicates that a node with Compress op will not be pushed twice. Consider if the helpMove function detects op.iParent.op as Compress, then op.iParent is not reachable in the next time. Hence, quadtree is stabilized and only when flag $\rightarrow$ unflag transitions execute infinitely and never set ${ }_{0} C A S$ as true.

According to Lemma 46, we order spatial order on both op.iParent and op.rParent as $i p_{0}>i p_{1} \ldots>$ $i p_{n}$ and $r p_{0}>r p_{1} \ldots>r p_{n}$. We assume that there's a ring such that move $e_{i}$ which flags $r p_{i}$ but fails on $i p_{i}$, and $m o v e_{i}^{\prime}$ which flags $i p_{i}$ but fails on $r p_{i}$. By assumption we order $r p_{i}$ and $i p_{i}$ as $r p_{i}>i p_{i}$ by move $_{i}$ 's order, and $i p_{i}>r p_{i}$ by move $e_{i}^{\prime \prime}$ s order. But by Lemma 46 , all Internal nodes in a snapshot can be ordered uniquely. Therefore it derives a contradiction.

Then, we prove that there's no ring among all move $_{i}$ in the same snapshot, so at least one of move $_{i}$ could set $d o C A S$ to true, resulting in the flag $\rightarrow$ replace $\rightarrow$ unflag transition. Let's consider the dependency among all move $e_{i}$. If move $e_{i}$ which fails on $p_{i}$ has been flagged by move $_{i}^{\prime}$, then there is a directed edge from move ${ }_{i}$ to move $_{i}^{\prime}$ by order. In this way, the head node in the graph must have no out edge as proved. It will set $d o C A S$ to true, all move operations that are directly connected to it will help it finish. After erase the former head node from the graph, there will be other nodes that have no out edge. Finally, all dependency edges are removed. Therefore, there are a finite number of flag $\rightarrow$ unflag transitions.

Observation 8. The help function, the helpCompress function, the helpMove function, and the helpSubstitute function are not called in a mutual way. (If method A calls method $B$, and method $B$ calls method $A$ reciprocally, we say method $A$ and $B$ are called in a mutual way.)

Lemma 48. If help function returned at $T_{i}$, and find(keys) at the prior iteration reads $p^{0}$.op at $T_{i 1}<T_{i}$, Leaf nodes in snapshot $_{T_{i}}$ and snapshot $T_{T_{i 1}}$ are different.

Proof. We prove the lemma by contradiction, there are two 
possible scenarios. First, the help function might be interminable. But based on Observation 8 , the help function must terminate. Second, the help function might execute without changing quadtree. According to Lemma 47 there are finite number of CAS transitions. So if some help function does not change quadtree, the structure might be changed before $T_{i 1}$. For the move function, if $i p$ and $r p$ are the same but their ops are different, the help function might not change the structure. Nevertheless, snapshot $T_{T_{i}}$ and snapshot $_{T_{i 1}}$ are different. Or else, there are some interval that all transitions are flag $\rightarrow$ unflag. However, Lemma 47 also shows that all transitions form a acyclic graph that there is no interval that all transitions are flag $\rightarrow$ unflag. Therefore, a flag $\rightarrow$ replace $\rightarrow$ unflag transition or a flag $\rightarrow$ unflag transition is executed to change the snapshot.

Lemma 49. Our quadboost algorithms are non-blocking.

Proof. We have to prove that no process will execute loops infinitely without changing keys in quadtree. First, we prove that path is terminable. Next, we prove that $\operatorname{find}\left(k_{e y s}{ }^{k}\right)$ starts from an active node in physical_path $\left(\right.$ keys $\left.^{k}\right)$ in snapshot $_{T_{i}}$ between $i_{t h}$ iteration and $i+1_{t h}$ iteration. Finally, Leaf nodes in snapshot $T_{i 1}$ at the returning of find(keys) at $i_{\text {th }}$ iteration is different from snapshot $_{T_{i 2}}$ that at the returning of $f i n d(k e y s)$ at the $i+1_{t h}$ iteration.

For the first part, initially we start from the root node. Therefore, path is empty. Moreover, as Lemma 45 shows that path $^{k}$ consists of finite number of keys, we establish this part.

For the second part, the continueFind function and the continueFindCommon function pop all nodes with a Compress op from path. For the insert operation and the remove operation, since Lemma 29 shows that an Internal nodes whose $o p$ is not Compress is active, and Lemma 31 shows that nodes above the active node are also active, there is a snapshot in which the top node of path is still in physical_path $\left(k_{e y s}{ }^{k}\right)$. For the move operation, if either rFail or $i F a i l$ is true, it is equivalent with the prior case. If $c$ Fail is true, Lemma 32 illustrates that if the LCA node is active, it is in physical_path for both oldKey and newKey. Thus, there is also a snapshot in which the start node is in physical_path.

We prove the third part by contradiction, assuming that quadtree is stabilized at $T_{i}$, and all invocations after $T_{i}$ are looping infinitely without changing Leaf nodes.

For the insert and remove operations, before the invocation of $f$ ind(keys) at the next iteration, they must execute the help function at line 118 and line 175 accordingly. In both cases, the help function changes the snapshot (Lemma 48.

For the move operation, consider different situations of the continueFindCommon function. If $r$ Fail or $i$ Fail is true, two situations arise: (1) $i O p$ or $r O p$ is Clean but $m$ flag fails. (2) $i O p$ or $r O p$ is not Clean. Consider the first case that $m$ flag fails, $i O p$ and $r O p$ are updated respectively at line 236 and line 235 If ip and $r p$ are different, before its invocation of $\operatorname{find}\left(k_{e y s} s^{k}\right)$, the help function is performed at line 311 and line 287 Thus by Lemma 48, the snapshot is changed between two iterations. Now consider if cFail is true. If $i p$ and $r p$ are the same, it could result from the difference between $r O p$ and $i O p$. In this case, the snapshot might be changed between reading $r O p$ and $i O p$. It could also result from the failure of mflag. For above cases, the help function at line 332 would change quadtree. If $i p$ and $r p$ are different, it results from that either $i$ Path or $r$ Path has popped the LCA node. We have proved the case in the former paragraph. Thus, we derives a contradiction.

From above discussions, we prove that quadboost is non-blocking. 